\title{
Two homologs of the Cat8 transcription factor are involved in the regulation of ethanol utilization in Komagataella phaffii
}

\author{
Diane Barbay $^{1,2} \cdot$ Monika Mačáková $^{2} \cdot$ Leander Sützl $^{3} \cdot$ Sonakshi De $^{1,2} \cdot$ Diethard Mattanovich $^{1,2}$ (D) \\ Brigitte Gasser ${ }^{1,2}$ (D)
}

Received: 6 August 2020 / Revised: 17 February 2021 / Accepted: 18 February 2021 / Published online: 16 March 2021

(c) The Author(s) 2021

\begin{abstract}
The transcription factors Cat8 and Sip4 were described in Saccharomyces cerevisiae and Kluyveromyces lactis to have very similar DNA binding domains and to be necessary for derepression of a variety of genes under non-fermentative growth conditions via binding to the carbon source responsive elements (CSREs). The methylotrophic yeast Komagataella phaffi (syn Pichia pastoris) has two transcription factors (TFs), which are putative homologs of Cat8 based on sequence similarity, termed Cat8-1 and Cat8-2. It is yet unclear in which cellular processes they are involved and if one of them is actually the homolog of Sip4. To study the roles of the Cat8 homologs in K. phaffii, overexpression or deletion strains were generated for the two TFs. The ability of these mutant strains to grow on different carbon sources was tested, and transcript levels of selected genes from the carbon metabolism were quantified. Our experiments showed that the TFs are required for the growth of $K$. phaffii on $\mathrm{C} 2$ carbon sources, but not on glucose, glycerol or methanol. K. phaffii deleted for Cat8-1 showed impaired growth on acetate, while both Cat8-1 and Cat8-2 are involved in the growth of $K$. phaffii on ethanol. Correspondingly, both TFs are participating in the activation of $A D H 2, A L D 4$ and $A C S 1$, three genes encoding enzymes important for the assimilation of ethanol. Different from $S$. cerevisiae and K. lactis, Cat8-1 is not regulating the transcription of the putative Sip4family member Cat8-2 in K. phaffii. Furthermore, Cat8-1 is necessary for the activation of genes from the glyoxylate cycle, whereas Cat8-2 is necessary for the activation of genes from the carnitine shuttle. Neither Cat8-1 nor Cat8-2 are required for the activation of gluconeogenesis genes. Finally, the CAT8-2 gene is repressed by the Mig1-2 transcription factor on glucose and autorepressed by the Cat8-2 protein on all tested carbon sources. Our study identified the involvement of $K$. phaffi Cat8-1 and Cat8-2 in C2-metabolism, and highlighted similarities and differences to their homologs in other yeast species.
\end{abstract}

Keywords Komagataella phaffi $\cdot$ Pichia pastoris $\cdot$ Yeast $\cdot$ Cat8 $\cdot$ Sip4 $\cdot$ CSRE $\cdot$ Transcription factor $\cdot$ Ethanol utilization $\cdot$ Carbon source

Communicated by Michael Polymenis.

Brigitte Gasser

brigitte.gasser@boku.ac.at

1 Austrian Centre of Industrial Biotechnology (ACIB), Vienna, Austria

2 Department of Biotechnology, Institute of Microbiology and Microbial Biotechnology, University of Natural Resources and Life Sciences (BOKU), Vienna, Austria

3 Department of Food Technology, University of Natural Resources and Life Sciences (BOKU), Vienna, Austria

\section{Introduction}

The yeast Komagataella phaffi (Pichia pastoris) adapts to different growth conditions through various mechanisms, including reprogramming of gene expression and protein synthesis (Hartner and Glieder 2006; Lin-Cereghino et al. 2006; Prielhofer et al. 2015). The release from glucose and catabolite repression alters the transcription of genes involved in numerous cellular processes, such as glycolysis, gluconeogenesis, tricarboxylic acid (TCA) cycle, and metabolism of alternative carbon sources (Prielhofer et al. 2015). Growth on gluconeogenic carbon sources such as glycerol and ethanol requires enzymes from gluconeogenesis and the glyoxylate cycle, among others. In the yeasts Saccharomyces cerevisiae and Kluyveromyces lactis, the genes 
encoding these enzymes are activated through upstream activation sites (UAS) found in their promoters, such as the carbon source responsive elements (CSREs) (Mehlgarten et al. 2015; Turcotte et al. 2010). The CSREs are under the control of two transcriptional regulators, which are members of the binuclear zinc cluster family: Cat8 and Sip4 (Roth et al. 2004; Vincent and Carlson 1998).

Cat8 (CATabolite repression) and Sip4 (Snf1 interacting protein) possess a highly similar $\mathrm{N}$-terminal zinc cluster $\left(\mathrm{Zn}(\mathrm{II})_{2} \mathrm{Cys}_{6}\right)$ binding domain (Rahner et al. 1996), but they share only little similarity in the rest of their protein sequences (Mehlgarten et al. 2015; Turcotte et al. 2010). Although both Cat8 and Sip4 were shown to bind the CSRE (consensus sequence: YCCRTTNRNCGG) (Roth et al. 2004; Vincent and Carlson 1998), Sip4 recognizes and binds to a more specific CSRE motif than Cat8, which probably explains why Cat8 and Sip4 contribute unequally to gene activation via binding to this motif (Hiesinger et al. 2001). In S. cerevisiae and $K$. lactis, Cat8 and Sip4 were described to be activators of transcription, but their mechanism of action in these two yeast are slightly different (Mehlgarten et al. 2015).

In $S$. cerevisiae, the expression and activities of CAT8 ( $S c \mathrm{Cat} 8)$ and SIP4 (ScSip4) were shown to be regulated by glucose, in a process mediated by the Snf1 kinase (Hardie et al. 1998). This kinase has a fundamental role in glucose derepression through the activation of various transcriptional activators and the deactivation of the $\mathrm{Cys}_{2} \mathrm{His}_{2}$ zinc finger protein Mig1. In the presence of glucose, the transcription factor (TF) Mig1 binds to the promoter of genes such as CAT8, which represses their expression. On derepressing conditions, the Snf1 kinase phosphorylates Mig1, which leads to its inactivation and the consequent induction of CAT8 transcription (Carlson 1999; Schüller 2003). The Cat 8 protein is then activated via phosphorylation by Snf1, and induces the transcription of various genes involved in the growth on non-fermentable carbon sources such as genes of the $\mathrm{C} 2$ anabolism, the glyoxylate cycle, and gluconeogenesis (De Vit et al. 1997; Haurie et al. 2001; Hedges et al. 1995; Lesage et al. 1996; Randez-Gil et al. 1997; Tachibana et al. 2005). Sccat8 knock-out mutants are unable to grow on gluconeogenic carbon sources such as glycerol, ethanol, lactate, and acetate (Hedges et al. 1995; Rahner et al. 1996), whereas Scsip4 knock-out mutants have no apparent growth phenotype on any of the tested carbon sources. This indicates that in S. cerevisiae, Sip4 plays a minor role in CSREdependent regulation (Lesage et al. 1996). In addition, the SIP4 promoter contains CSRE motifs which were shown to be bound by Cat 8 , indicating that Cat 8 is regulating the transcription of SIP4 in S. cerevisiae (Vincent and Carlson, 1998).

In the yeast $K$. lactis, both Cat8 (KlCat8) and Sip4 (KlSip4) are present but the regulatory networks are different than in S. cerevisiae. The Klcat8 knock-out mutants can grow on glycerol but not on $\mathrm{C} 2$ carbon sources such as acetate and ethanol, which shows that unlike in S. cerevisiae, the gluconeogenesis encoding genes are not regulated by Cat 8 nor Sip4 in K. lactis (Georis et al. 2000). On the other hand, contrary to $S$. cerevisiae where Cat 8 but not Sip4 is required for growth on ethanol, both Klcat8 and Klsip4 knock-out mutants exhibit a growth defect on $\mathrm{C} 2$ carbon sources (Mehlgarten et al. 2015). It was also shown that only KlSip4 binds to the CSRE motifs in the promoters of the glyoxylate pathway genes and the carnitine shuttle encoding genes (Mehlgarten et al. 2015; Rodicio et al. 2008). In $K$. lactis, as in S. cerevisiae, Cat8 was shown to activate the transcription of Sip4. KlCat8 was also shown to be regulated via phosphorylation of a conserved serine residue (Ser-661) by the Snf1 kinase (Charbon et al. 2004).

Homologs of the Cat8 transcription factor were also found in other yeasts, although their function was not studied in as much depth as in S. cerevisiae or K. lactis. Candida albicans Cat8 (CaCat8) knock-out mutants have a similar phenotype to the wild-type in terms of gluconeogenesis, glyoxylate shunt, and ethanol utilization pathway, and $\mathrm{CaCat} 8$ does not seem to regulate the gluconeogenic gene $P C K 1$ nor $I C L 1$ (encoding isocitrate lyase, an enzyme of the glyoxylate cycle) (Ramirez and Lorenz 2009). Cat8 knock-out mutants in Ogataea (Hansenula) polymorpha exhibit a growth defect on glycerol, ethanol, and xylose, and have a higher ethanol production from xylose fermentation (Ruchala et al. 2017). Finally, in Pichia guillermondii, knocking-out the CAT8 gene triggers respiro-fermentative metabolism of this Crabtree-negative yeast (Qi et al. 2014).

In Komagataella phaffii, two putative homologs of Cat8 termed CAT8-1 (CBS7435 locus name PP7435_Chr2-0516) and CAT8-2 (PP7435_Chr4-0434) are found. The CAT8-2 gene was shown to be induced on limiting glucose (about 39-fold up-regulated compared to excess glucose) and on methanol (about sevenfold up-regulated compared to excess glucose), indicating that it is subject to glucose repression. No up- or down-regulation of CAT8- 1 was observed in the same conditions (Prielhofer et al. 2015). It is yet unclear which genes are regulated by the TFs Cat $8-1$ and Cat $8-2$ and if one of them is the homolog of Sip4. In order to study the role of CAT8- 1 and CAT8-2 in K. phaffii, overexpression and knock-out mutants of each of these two TF genes were generated and the ability of the TF mutant strains to grow on different nutrient sources were tested. Based on the findings, the transcript levels of some selected genes from the carbon metabolism were quantified to see how their regulation patterns on different carbon sources were affected in the absence of either or both Cat8 homologs. 


\section{Materials and methods}

\section{Strains, primers and plasmids}

All the Komagataella phaffii strains used in this study (Table 1) were derived from the wild-type strain CBS7435. Escherichia coli DH10B (Invitrogen) were used for cloning experiments.

\section{Sequence analyses, alignment and phylogenetic tree}

Eight individual protein-BLAST searches were conducted on the 'Non-redundant protein sequences (nr)' database of NCBI using the following 6 functionally characterized protein sequences: CBF88979.1, Cat8 of Aspergillus nidulans (Todd et al. 1997, 1998); XP_018209149.1, Cat8 of Ogataea polymorpha (Ruchala et al. 2017); XP_453133.1/ AAC23607.1, Cat8 of $K$. lactis (Georis et al. 2000); CAE00852.1, Sip4 of K. lactis (Mehlgarten et al. 2015); CAA55139.1, Cat8 of $S$. cerevisiae (Hedges et al. 1995) and CAA89382.1, Sip4 of S. cerevisiae (Lesage et al. 1996) along with XP_002491690.1 and XP_002493979.1, Cat8-1 and Cat8-2 of $K$. phaffi, given here with their NCBI identifiers. For the two $K$. phaffii proteins, the entries of the reference sequence of $K$. phaffii GS115 were taken, which are identical to the protein sequences in $K$. phaffii CBS7435 used for the experiments in this study. The search was restricted to Saccharomycetes (Yeast; taxid: 4891) and a maximum $E$-value of $9 \mathrm{e}-30$.

All individual BLAST search results were combined and protein ID duplicates were removed (361 sequences). Then, all sequences containing invalid protein characters (B J O $\mathrm{U} X \mathrm{X}$ ) were removed from the selection (352 sequences), remaining sequences were filtered for a minimum length of 600 amino acids (275 sequences) and sequences in the selection of $99 \%$ sequence identity or higher were represented in the selection by only one sequence of that cluster to reduce sequence redundancy (157 sequences). Together with all characterized Cat8 and Sip4 sequences this resulted in a selection of 161 sequences.

The selection was aligned with MAFFT G-INS-I (Katoh and Standley 2013) and renamed with SeqScrub (Foley et al. 2019) according to taxonomy. The alignment was trimmed for positions with $>90 \%$ gaps by trimAl (CapellaGutierrez et al. 2009) and a maximum likelihood tree was calculated with PhyML (Guindon et al. 2010) using the LG amino acid substitution model, the best of NNI and SPRs moves to optimize tree topology and SH-like branch
Table 1 List of $K$. phaffii strains used in this study

\begin{tabular}{|c|c|c|c|c|}
\hline Name & Target (ORF name) & Target (short name) & $\begin{array}{l}\text { Target type } \\
\text { (OE/KO/ } \\
\text { Prom) }\end{array}$ & Source \\
\hline cat $8-2 \Delta$ & PP7435_Chr4-0434 & CAT8-2 & $\mathrm{KO}$ & This study \\
\hline cat $8-1 \Delta$ & PP7435_Chr2-0516 & CAT8-1 & KO & This study \\
\hline CAT8-2_OE & PP7435_Chr4-0434 & CAT8-2 & $\mathrm{OE}$ & This study \\
\hline CAT8-1_OE & PP7435_Chr2-0516 & CAT8-1 & $\mathrm{OE}$ & This study \\
\hline 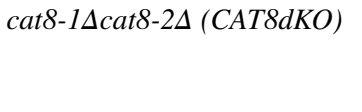 & $\begin{array}{l}\text { PP7435_Chr2-0516 } \\
\text { / PP7435_Chr4- } \\
0434\end{array}$ & CAT8-1/CAT8-2 & KO & This study \\
\hline CAT8-1_HAtag & PP7435_Chr2-0516 & CAT8-1 & TAG & This study \\
\hline CAT8-2_HAtag & PP7435_Chr4-0434 & CAT8-2 & TAG & This study \\
\hline pCAT8-2_eGFP_CAT8-1KO & PP7435_Chr4-0434 & CAT8-2 & Prom & This study \\
\hline pCAT8-2_eGFP_CAT8-2KO & PP7435_Chr4-0434 & CAT8-2 & Prom & This study \\
\hline pCAT8-2_eGFP_WT & PP7435_Chr4-0434 & CAT8-2 & Prom & This study \\
\hline pCAT8-2_eGFP_CAT8dKO & PP7435_Chr4-0434 & CAT8-2 & Prom & This study \\
\hline pCAT8-1_eGFP_WT & PP7435_Chr2-0516 & CAT8-1 & Prom & This study \\
\hline pCAT8-1_eGFP_CAT8-1KO & PP7435_Chr2-0516 & CAT8-1 & Prom & This study \\
\hline pCAT8-1_eGFP_CAT8-2KO & PP7435_Chr2-0516 & CAT8-1 & Prom & This study \\
\hline pCAT8-1_eGFP_CAT8dKO & PP7435_Chr2-0516 & CAT8-1 & Prom & This study \\
\hline 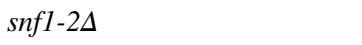 & PP7435_Chr1-0450 & SNF1-2 & KO & This study \\
\hline $\operatorname{ssn} 3 \Delta$ & PP7435_Chr1-1091 & SSN3 & $\mathrm{KO}$ & This study \\
\hline mig1-1s & PP7435_Chr4-0661 & $M I G 1-1$ & $\mathrm{KO}$ & Ata et al. (2017) \\
\hline MIG1-1_OE & PP7435_Chr4-0661 & $M I G 1-1$ & $\mathrm{OE}$ & Ata et al. (2017) \\
\hline mig $1-2 \Delta$ & PP7435_Chr1-1325 & MIG1-2 & KO & Ata et al. (2017) \\
\hline MIG1-2_OE & PP7435_Chr1-1325 & MIG1-2 & $\mathrm{OE}$ & Ata et al. (2017) \\
\hline
\end{tabular}


support. The tree was rooted on midpoint. All protein identifiers (NCBI accession numbers) of sequences used in the alignment are listed in Supplementary material 1.

\section{Prediction of transcription factor binding sites (TFBS)}

The regions upstream of all $K$. phaffi i genes (1000 bps upstream of the start codon) were analyzed for the occurrence of the CSRE by MatInspector (matrix F\$CSRE). Comparative analysis of TFBS in S. cerevisiae, K. lactis and $K$. phaffii promoters was done by Yeastract+ (http:// www.yeasttract-plus.org; Monteiro et al. 2020).

\section{Media}

YPD medium contained $10 \mathrm{~g} / \mathrm{L}$ yeast extract, $20 \mathrm{~g} / \mathrm{L}$ soy peptone and $2 \%$ glucose as carbon source. YPD agar plates consisted of $10 \mathrm{~g} / \mathrm{L}$ yeast extract, $20 \mathrm{~g} / \mathrm{L}$ soy peptone, $2 \%$ glucose as carbon source and $20 \mathrm{~g} / \mathrm{L}$ agar-agar. The YPD liquid medium and the YPD agar plates were supplemented with the appropriate antibiotics (zeocin $50 \mu \mathrm{g} /$ $\mathrm{mL}$, geneticin $500 \mu \mathrm{g} / \mathrm{mL}$, nourseothricin $100 \mu \mathrm{g} / \mathrm{mL}$ ) when needed.

LB media consisted of $10 \mathrm{~g} / \mathrm{L}$ soy peptone, $5 \mathrm{~g} / \mathrm{L}$ yeast extract, $5 \mathrm{~g} / \mathrm{L} \mathrm{NaCl} .20 \mathrm{~g} / \mathrm{L}$ agar-agar was added to prepare LB agar plates.

ASMv6 medium contained per liter $6.3 \mathrm{~g}\left(\mathrm{NH}_{4}\right)_{2} \mathrm{HPO}_{4}$,

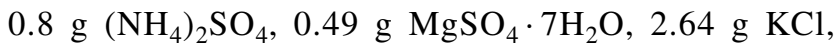
$0.0535 \mathrm{~g} \mathrm{CaCl}_{2} \cdot 2 \mathrm{H}_{2} \mathrm{O}, 22 \mathrm{~g}$ citric acid monohydrate, $1.47 \mathrm{~mL}$ PTM, $2 \mathrm{~mL}$ biotin $(0.2 \mathrm{~g} / \mathrm{L}), 20 \mathrm{~mL} \mathrm{NH}_{4} \mathrm{OH}(25 \%)$ with additional carbon source according to the purpose. For limited glucose condition 25\% m2p kit Polysaccharide and $0.078 \%$ enzyme was used ( $\mathrm{m} 2 \mathrm{p}$-labs $\mathrm{GmbH}$, Germany).

PTM0 stock solution contained per liter $0.08 \mathrm{~g} \mathrm{NaI}, 6.0 \mathrm{~g}$ $\mathrm{CuSO}_{4} \cdot 5 \mathrm{H}_{2} \mathrm{O}, 3.36 \mathrm{~g} \mathrm{MnSO}_{4} \cdot \mathrm{H}_{2} \mathrm{O}, 0.2 \mathrm{~g} \mathrm{Na}_{2} \mathrm{MoO}_{4} \cdot 2 \mathrm{H}_{2} \mathrm{O}$, $0.82 \mathrm{~g} \mathrm{CoCl}_{2}, 0.02 \mathrm{~g} \mathrm{H}_{3} \mathrm{BO}_{3}, 20.0 \mathrm{~g} \mathrm{ZnCl}_{2}, 65.0 \mathrm{~g}$ $\mathrm{FeSO}_{4} \cdot 7 \mathrm{H}_{2} \mathrm{O}$ and $5.0 \mathrm{~g} \mathrm{~mL} \mathrm{H}_{2} \mathrm{SO}_{4}(95-98 \%)$.

YNB without thiamine consisted of $10 \mathrm{~g} / \mathrm{L}\left(\mathrm{NH}_{4}\right)_{2} \mathrm{SO}_{4}$, $0.2 \mathrm{~g} / \mathrm{L}$ biotin, $0.8 \mathrm{mg} / \mathrm{L}$ Ca-pantothenate, $0.004 \mathrm{mg} / \mathrm{L}$ folic acid, $4 \mathrm{mg} / \mathrm{L}$ inositol, $0.8 \mathrm{mg} / \mathrm{L}$ niacin, $0.4 \mathrm{mg} / \mathrm{L}$ p-aminobenzoic acid, $0.8 \mathrm{mg} / \mathrm{L}$ pyridoxine $\mathrm{HCl}, 0.4 \mathrm{mg} / \mathrm{L}$ riboflavin, $1 \mathrm{mg} / \mathrm{L} \mathrm{H}_{3} \mathrm{BO}_{3}, 0.08 \mathrm{mg} / \mathrm{L} \mathrm{CuSO}_{4}, 0.2 \mathrm{mg} / \mathrm{L}$ $\mathrm{KI}, 0.4 \mathrm{mg} / \mathrm{L} \mathrm{FeCl}_{3}, 0.8 \mathrm{mg} / \mathrm{L} \mathrm{MnSO}_{4} \cdot \mathrm{H}_{2} \mathrm{O}, 0.4 \mathrm{mg} / \mathrm{L}$ $\mathrm{Na}_{2} \mathrm{MoO}_{4} \cdot 2 \mathrm{H}_{2} \mathrm{O}, 0.8 \mathrm{mg} / \mathrm{L} \mathrm{ZnSO}_{4}, 2 \mathrm{~g} / \mathrm{L} \mathrm{KH}_{2} \mathrm{PO}_{4}, 1 \mathrm{~g} / \mathrm{L}$ $\mathrm{MgSO}_{4}, 200 \mathrm{mg} / \mathrm{L} \mathrm{NaCl}, 200 \mathrm{mg} / \mathrm{L} \mathrm{CaCl}_{2}$ with additional carbon source according to the purpose. For the limiting glucose condition 25\% m2p kit Polysaccharide and $0.078 \%$ enzyme was used (m2p-labs GmbH, Germany).

Phosphate buffered saline (PBS) consisted of $1.8 \mathrm{~g} / \mathrm{L}$ $\mathrm{Na}_{2} \mathrm{HPO}_{4} \cdot 2 \mathrm{H}_{2} \mathrm{O}, 0.24 \mathrm{~g} / \mathrm{L} \mathrm{KH}_{2} \mathrm{PO}_{4}, 8 \mathrm{~g} / \mathrm{L} \mathrm{NaCl}, 0.2 \mathrm{~g} / \mathrm{L}$ $\mathrm{KCl}$.

\section{Genomic DNA extraction and PCR}

Genomic DNA was extracted from overnight cultures using the Wizard genomic DNA purification kit (Promega Corp., USA) according to the protocol of the manufacturer. All PCRs were performed using the Q5 polymerase (New England Biolabs, Inc., USA) following the recommendations of the manufacturer or using the OneTaq $2 \times$ master mix with GC buffer (New England Biolabs, Inc., USA). The PCRs were used to amplify DNA for cloning and for verifying positive transformants.

\section{Construction of overexpression and knock-out strains}

The K. phaffii CBS7435 chromosomal regions of the selected transcription factors are: PP7435_Chr2-0516 for CAT8-1 and PP7435_Chr4-0434 for CAT8-2. The sequences of these genes were retrieved from http://pichi agenome-ext.boku.ac.at.

Golden Gate Assembly (GGA; Engler et al. 2008) was used for the construction of the overexpression and knockout cassettes using the Golden $P i C S$ vector series (Prielhofer et al. 2017). Internal $B s a \mathrm{I}$ or $B p i \mathrm{I}$ sites within the CDS or homologous regions for the integration of knock-out cassettes were eliminated by designing primers which enabled to overlap the modified regions by PCR or ordering in vitro synthesized gBlocks where these nucleotides were mutated without altering the originally encoded amino acids.

\section{Overexpression}

For overexpression cassettes, the CDS of the corresponding genes were amplified from the $K$. phaffii CBS7435 genome by PCR and cloned into a plasmid carrying the KanMX marker cassette and a region for homologous integration into the AOXI terminator. The THIII promoter, the promoter of a gene which encodes a protein involved in the synthesis of the thiamine precursor hydroxymethyl pyrimidine (HMP), was used for the overexpression of selected TFs (Delic et al. 2013). The expression capacity of this promoter can be controlled by the presence or absence of thiamine (Landes et al. 2016). For all overexpression cassettes, the RPS3tt transcription terminator was used. Primers used for the generation of overexpression cassettes are given in Supplementary table $\mathrm{S} 1$. The transformants were verified by colony PCR and gene copy number determination (positive clones had two copies of the gene of interest: the native one and the one of the overexpression cassette). 


\section{Knock-outs}

Komagataella phaffi knock-out strains were constructed by using CRISPR/Cas9-based homology-directed genome editing (Gassler et al. 2019). The homologous regions (HR) were amplified by PCR from the K. phaffii CBS7435 genome. These homologous regions were selected from upstream $\left(5^{\prime}\right)$ and downstream ( $\left.3^{\prime}\right)$ of the target gene with an approximate $1000 \mathrm{bp}$ length. The primers used for amplification of these homologous fragments and elimination of internal BsaI sites are given in Supplementary table S2. The flanking upstream and downstream homologous regions of the target gene were assembled with each other in a plasmid by GGA.

A single guide RNA was designed and amplified based on a protospacer adjacent motif (PAM) sequence identified in 50-200 bp upstream of the CDS of the selected TFs [see Gassler et al. (2019) for more details on the construction of the single guide RNA]. The guide RNA was cloned under the control of the GAP promoter and the RPS25Att terminator into a plasmid containing the humanized Cas9 CDS under the control of $\mathrm{P}_{\mathrm{LAT} 1}$ or $\mathrm{P}_{\mathrm{PFK} 300}$ and the ScCYCtt terminator by GGA. For the integration of the knock-out cassettes, the plasmids carrying the fused homologous regions were used as templates to amplify the fragments by PCR. 3-5 $\mu \mathrm{g}$ of amplified homologous DNA and $0.5-1 \mu \mathrm{g}$ of circular CRISPR/Cas9 plasmid DNA were simultaneously transformed into K. phaffii CBS7435 by electroporation (Gasser et al. 2013). The knock-out (KO) strains were checked by two PCRs using (1) primers binding in the genome outside of the targeted deletion sites and (2) binding in the CDS of the targeted TFs. After confirmation of the TF deletions, true KO transformants were passaged at least three times on YPD to lose the CRISPR/Cas9 plasmid.

\section{Transformation of K. phaffii}

Prior to K. phaffii transformation by electroporation (Gasser et al. 2013), the overexpression plasmids were linearized within the genome integration locus by AscI and purified (innuPREP DOUBLEpure Kit, Analytik Jena, Germany). PCR-amplified homologous regions of knock-out cassettes were directly purified without linearizing. $K$. phaffii transformation was performed by electroporation (BioRad Gene Pulser, $2000 \mathrm{~V}, 25 \mu \mathrm{F}$ and $200 \Omega$ ) by using $0.5-1 \mu \mathrm{g}$ of each linearized overexpression plasmid or 3-5 $\mu \mathrm{g}$ of purified knock-out fragments and $0.5-1 \mu \mathrm{g}$ of circular CRISPR/Cas9 plasmid. Transformed cells were then regenerated by incubation at $30^{\circ} \mathrm{C}$ for $1.5-3 \mathrm{~h}$ in YPD medium $(280 \mathrm{rpm})$ and then plated on YPD plates including the appropriate antibiotics concentration (zeocin $50 \mu \mathrm{g} / \mathrm{mL}$, geneticin $500 \mu \mathrm{g} /$ $\mathrm{mL}$, nourseothricin $100 \mu \mathrm{g} / \mathrm{mL}$ ). After $48-72 \mathrm{~h}$ at $30{ }^{\circ} \mathrm{C}$, randomly selected transformants were streaked on selective YPD plates and incubated $48 \mathrm{~h}$ at $30^{\circ} \mathrm{C}$.

\section{Gene copy number determination}

Gene copy number (GCN) was determined by quantitative real-time PCR (qRT-PCR). Genomic DNA was extracted from overnight cultures using the Wizard genomic DNA purification kit (Promega Corp., USA). The GCN was determined by the relative quantification of the TF of interest sequence compared to wild-type $K$. phaffii CBS7435 (carrying a single native copy of the $\mathrm{TF}$ gene of interest). The amplifications were carried out using $4.5 \mu \mathrm{L}$ of genomic DNA solution at a concentration of $1.777 \mathrm{ng} / \mu \mathrm{L}$ with 0.25 $\mu \mathrm{L}$ of both forward and reverse primers (final concentration: $10 \mu \mathrm{M}$ ) and $5 \mu \mathrm{L}$ of $2 \times \mathrm{qPCR}$ S'Green BlueMix (Biozym Scientific GmbH, Germany). Amplifications were done in a Rotor-Gene Q instrument (QIAGEN GmbH, Germany). The GCN in the K. phaffii mutant strains were calculated relative to the corresponding wild-type control using the threshold cycle $(\triangle \triangle C T)$ method. All signals were normalized to ACTI (PP7435_Chr3-0993). The primers used for qRT-PCR analysis are provided in Supplementary table S3.

\section{Growth assays in liquid medium}

The wild-type, overexpression and deletion strains were inoculated at $\mathrm{OD}_{600} 0.01$ in $100 \mu \mathrm{L}$ of $\mathrm{YNB}$ without thiamine containing either $2 \%$ glucose, $2 \%$ glycerol, $2 \%$ ethanol or $1 \%$ methanol in a 96 -well sterile microtiter plate. Growth on acetate was assessed in $100 \mu \mathrm{L}$ of YNB or YP containing $1 \%$ or $2 \%$ acetate. The plate was incubated in a TECAN Sunrise plate reader at $30{ }^{\circ} \mathrm{C}$ for $24-48 \mathrm{~h}(99$ cycles, interval 14:39 $\mathrm{min}$ ) with constant shaking (before measurement $5 \mathrm{~s}$, inside, normal; between cycles $870 \mathrm{~s}$, inside, normal). The absorbance at $600 \mathrm{~nm}$ in each well was measured every $15 \mathrm{~min}$.

For each strain, three to four biological replicates were cultivated each in three wells. The blank value $\left(\mathrm{OD}_{600}\right.$ of the media without cells) was subtracted from the raw OD values to obtain the corrected ODs. The corrected $\mathrm{OD}_{600}$ of the replicates for a given strain were then averaged. The average corrected $\mathrm{OD}_{600}$ was plotted against the time to obtain the growth curves. For the calculation of the specific growth rates, the average corrected $\mathrm{OD}_{600}$ was divided by the average initial $\mathrm{OD}_{600}$ and the natural logarithm was applied. The growth rate is given by the slope of the log-transformed ODs, the maximal growth rate being identified as the maximum value of the slope (Toussaint and Conconi 2006). 


\section{RNA extraction and transcript levels analysis}

The wild-type, overexpression and deletion strains were grown on ASMv6 medium with limiting glucose to $\mathrm{OD}_{600}$ 7.0-8.0, washed twice in PBS, inoculated at $\mathrm{OD}_{600} 3.5-4.5$ in ASMv6 medium containing either $2 \%$ glucose, $2 \%$ glycerol, $2 \%$ ethanol or $1 \%$ methanol and grown for $5 \mathrm{~h}$, to have an induction on glucose, glycerol, ethanol, and methanol, respectively. Samples were then collected by centrifugation at full speed at $4{ }^{\circ} \mathrm{C}$, and cell pellets were resuspended in $1 \mathrm{~mL}$ TRI reagent solution (Invitrogen) and stored at $-70{ }^{\circ} \mathrm{C}$ until further use. Cells were mechanically disrupted using $500 \mu \mathrm{L}$ of glass beads in a ribolyzer $(5.5 \mathrm{~m} / \mathrm{s}$ for $40 \mathrm{~s})$, and the total RNA extraction was performed according to the TRI reagent protocol. RNA concentrations and purity were analyzed with a Nanodrop spectrophotometer. DNAse treatment of isolated RNA samples was performed with a DNA-free kit (Invitrogen) and cDNA was synthesized using oligo $(\mathrm{dT})_{23}$ primers (New England Biolabs, Inc., USA) and the Biozym cDNA synthesis kit according to directions of the manufacturer (Biozym Scientific GmbH, Germany). The Real-time PCR reactions were performed on a Rotor-Gene Q instrument (QIAGEN GmbH, Germany) using Blue S'Green qPCR Mix (Biozym Scientific GmbH, Germany) according to the manufacturer's instructions. Changes in transcript levels in the $K$. phaffii mutant strains were calculated relative to the corresponding wild-type control using the threshold cycle $(\Delta \Delta C T)$ method. All signals were normalized to the expression of the actin gene ACT1 (PP7435_Chr3-0993). The primers used for qRT-PCR analysis are provided in Supplementary table S4.

\section{Construction of the HA-tagged Cat8-1 and Cat8-2 strains}

Initially, the tagging of Cat8-1 and Cat8-2 was tested with a 3xFLAG tag at the native locus using the CRISPR/Cas9based homology-directed genome editing (Gassler et al. 2019), but no clones were obtained after transformation. Another approach was transforming plasmids containing the coding sequences of $C A T 8-1$ and $C A T 8-2$ with a $3 \times$ FLAG tag before their STOP codons under the control of their native promoters into K. phaffii cat8-1 $1 \Delta$ (for Cat8-1-3xFLAG) and cat8-2 $\Delta$ (for Cat8-2-3xFLAG) deletion strains. Again no clones were obtained. Since the single deletion strains of CAT8- 1 and CAT8-2 were viable, it seemed that introducing a FLAG-tagged copy of Cat8-1 and Cat8-2 was detrimental to the cells. Therefore tagging of Cat8-1 and Cat8-2 with the HA tag was tested, and was proven to be a successful strategy yielding viable transformants.

To construct the HA-tagged Cat8-1 and Cat8-2 strains, promoter regions (upstream $1000 \mathrm{bp}$ regions), coding sequences, and terminator regions of CAT8- 1 and CAT8-2 were amplified from $K$. phaffii CBS7435 genomic DNA by PCR. Reverse primers for the amplification on the coding sequences were designed so there is insertion of the HA tag (encoded by TACCCATACGATGTTCCAGATTAC GC) before the STOP codons of the two genes. The PCR fragments were assembled by GGA in a vector carrying the $A O X 1$ terminator homologous regions (for integration into the $K$. phaffii genome) and the KanMX marker cassette. 0.5-1 $\mu \mathrm{g}$ of linearized plasmids were transformed into

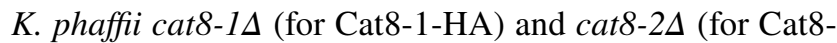
2-HA) deletion strains. Transformants were controlled by colony PCR and qRT-PCR (for gene copy number determination). The selected clones were also grown on YNB liquid medium supplemented with $1 \%$ ethanol to make sure that the insertion of the tagged version of the proteins in the deletion strains restored their growth on this carbon source.

\section{eGFP reporter assay}

\section{Cloning of eGFP under the control of $\mathrm{P}_{\text {CAT8-1 }}$ and $\mathrm{P}_{\text {CAT8-2 }}$}

Promoter regions (upstream $1000 \mathrm{bp}$ regions) of $C A T 8-1$ and CAT8-2 were amplified from $K$. phaffii CBS7435 genomic DNA by PCR (Primers in Supplementary table S5). The respective promoters, the eGFP coding sequence and the $S c C Y C t$ t were assembled by GGA in a vector containing $A O X 1$ terminator homologous regions (for integration into $K$. phaffi genome) and the KanMX marker cassette. $0.5-1 \mu \mathrm{g}$ of the linearized plasmids were transformed into $K$. phaf-

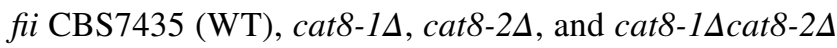
deletion strains. Transformants were controlled by colony PCR and qRT-PCR for gene copy number determination.

\section{Reporter assay}

The wild-type and positive transformants of the wild-type, cat $8-1 \Delta$, cat $8-2 \Delta$, and cat $8-1 \Delta$ cat $8-2 \Delta$ deletion strains carrying the eGFP coding sequence under the control of the respective promoters were grown on ASMv6 medium with limiting glucose (polysaccharide solution) at $25{ }^{\circ} \mathrm{C}$ to $\mathrm{OD}_{600}$ 7.0-8.0, washed twice in PBS and inoculated at $\mathrm{OD}_{600}$ 3.5-4.5 in ASMv6 medium containing either $2 \%$ glucose, $2 \%$ glycerol, $2 \%$ ethanol or $1 \%$ methanol and grown for $5 \mathrm{~h}$ at $25{ }^{\circ} \mathrm{C}$, to have an induction on glucose, glycerol, ethanol, and methanol, respectively. After induction, cells were analyzed by flow cytometry.

\section{Flow cytometry}

Cells were diluted in PBS $\left(\mathrm{KH}_{2} \mathrm{PO}_{4} 0.24 \mathrm{~g} / \mathrm{L}, \mathrm{Na}_{2} \mathrm{HPO}_{4} \cdot 2\right.$ $\mathrm{H}_{2} \mathrm{O} 1.8 \mathrm{~g} / \mathrm{L}, \mathrm{KCl} 0.2 \mathrm{~g} / \mathrm{L}, \mathrm{NaCl} 8 \mathrm{~g} / \mathrm{L}$ ) to $\mathrm{OD}_{600} 0.2$. The forward and side scatter of 10,000 cells for each sample as well as their green fluorescence (FL1 channel, 505-545 nm) 
were then measured on a CytoFLEX flow cytometer (Beckman Coulter). The Kaluza analysis software (Beckman Coulter) was used to analyze the data. GFP-positive cells were gated using the WT_P $\mathrm{PAT}_{\text {CA1-1_eGFP }}$ and WT_P $\mathrm{P}_{\text {CAT8-2_eGFP }}$ strains as reference.

\section{Results}

\section{Phylogenetic analysis of Cat8 and Sip4 homologs in Saccharomycetes}

The transcription factors Cat8 and Sip4 both belong to the family of binuclear zinc cluster proteins. These proteins are to date only found in fungi and typically possess a zinc cluster motif with the following consensus sequence: Cys $\mathrm{X}_{2} \mathrm{Cys}_{6} \mathrm{CysX}_{5-12} \mathrm{Cys}_{2} \mathrm{CysX}_{6-8}$ Cys. The six conserved cysteine residues are involved in the folding of the zinc cluster domain, which is important for DNA recognition. Within the DNA binding domain, a linker region connects the zinc cluster motif to a Leucine zipper-like dimerization domain, involved in protein-protein interactions. As usual in binuclear zinc cluster proteins, the DNA binding domain is located near the $\mathrm{N}$-terminus, while the activation domain is found at the C-terminus (Todd and Andrianopoulos 1997).

Based on reciprocal BLAST analysis, $K$. phaffii Cat8-1 and Cat8-2 were found to be two homologs of S. cerevisiae Cat8 (Valli et al. 2016). Both Cat8-1 and Cat8-2 exhibit the characteristic binuclear zinc cluster $\mathrm{Zn}(\mathrm{II})_{2} \mathrm{Cys}_{6}$ binding domain located at the $\mathrm{N}$-terminus. To find out if one of these two genes could encode a protein structurally related to Sip4, a phylogenic analysis was performed.

A total of 161 sequences from various yeasts including 6 functionally characterized Cat 8 and Sip4 protein sequences from S. cerevisiae (Cat8 1433 aa, Sip4 829 aa), K. lactis (Cat8 1445 aa, Sip4 717 aa), O. polymorpha (Cat8 942 aa) and A. nidulans (FacB 867 aa) as well as K. phaffii Cat8-1 (1036 aa) and Cat8-2 (887 aa) were aligned, and their phylogenic tree was calculated (Fig. 1).

Generally, a high sequence variation was observed within the Cat8 and Sip4 variants from different yeasts. Only the DNA binding domain was conserved in all sequences. Specifically, the six cysteine residues involved in forming this DNA binding domain are well conserved among the Cat8 and Sip4 homologs (Fig. 1A). The adjacent Leucine zipper dimerization domain in the $\mathrm{C}$-terminal region of the binuclear cluster is conserved as well (Fig. 1A). Apart from this, there is not much sequence similarity observed.

In the phylogenetic tree, we can observe a clear separation of Sip4 and Cat8 homologs and a further subseparation between Saccharomycetaceae and other yeast genera (Fig. 1B). Five major clades were identified according to functional annotation and fungal genus: the Cat $8-2$ clade of Phaffomycetaceae and Pichiaceae (containing K. phaffii Cat8-2), the Sip4 clade of Saccharomycetaceae and Saccharomycodaceae (containing the characterized Sip4 proteins from S. cerevisiae and K. lactis), the Cat8 clade of Saccharomycetaceae and Saccharomycodaceae (containing the characterized Cat8 proteins from $S$. cerevisiae and $K$. lactis), the Cat8 clade of Phaffomycetaceae and Pichiaceae (containing $K$. phaffii Cat8-1 and O. polymorpha Cat8), and finally the Cat8 clade of Debaryomycetaceae and Metschnikowiaceae. In addition, a small clade of 8 sequences of 5 different yeast genus and one Aspergillus Cat8 is also observed (Fig. 1B). A listing of species and sequences present in each clade is given in Supplementary material 1.

Based on this full-length protein sequences analysis, we can confirm that the Cat8-1 protein from $K$. phaffii clusters with other characterized Cat 8 proteins. The Cat8-2 protein from $K$. phaffii on the other hand clusters more closely with Sip4 proteins, although constituting a different clade than the characterized Sip4 homologs of S. cerevisiae and K. lactis (Fig. 1B).

\section{CAT8-1 and CAT8-2 are essential for ethanol assimilation in $K$. phaffii}

To identify in which cellular processes the TFs Cat8-1 and Cat8-2 are involved, overexpression and knock-out strains of CAT8-1 and CAT8-2 were generated. For the overexpression, both genes were cloned under the control of the tunable THII1 promoter, which is repressed in presence of thiamine in the growth medium and induced in thiamin-depleted conditions (Delic et al. 2013; Landes et al. 2016), and transformed in the wild-type $K$. phaffii strain CBS7435. The knock-out strains of CAT8-1 and CAT8-2 were generated using CRISPR/Cas9-based homology-directed genome editing (Gassler et al. 2019). The growth of the overexpression and knock-out strains, as well as the K. phaffii wild-type, was assessed in liquid cultures with YNB (without thiamine for the overexpression strains) containing either $2 \%$ glucose, $2 \%$ glycerol, $2 \%$ ethanol or $1 \%$ methanol (Fig. 2).

No significant difference was observed between the specific growth rates of the CAT8- 1 overexpression strains and the wild-type on the different carbon sources (Fig. 2A). For the CAT8-2 overexpression strains, there was no significant difference to the growth rates of the wild-type on glucose and methanol. However, the growth rate of the CAT8-2 overexpression strain was reduced on glycerol (Fig. 2A), and it reached the stationary phase later than the wild-type on this carbon source (Fig. 2B).

For the knock-out strains cat $8-1 \Delta$ and cat $8-2 \Delta$, no significant differences in the specific growth rates were observed compared to the ones of the wild-type on glucose, glycerol,

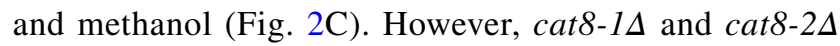
had significantly lower growth rates than the wild-type 


\section{A}

$\begin{array}{ll}\text { Cat8-2 } & \text { K. phaffi } \\ \text { Sip4 } & \text { K. lactis } \\ \text { Sip4 } & \text { S. cerevisiae } \\ \text { Cat8 } & \text { A. nidulans } \\ \text { Cat8 } & \text { S. cerevisiae } \\ \text { Cat8 } & \text { K. lactis } \\ \text { Cat8-1 } & \text { K. phaffi }\end{array}$

Cat8-1 K. phaffi
20 KFRI SQACDRCRIKKIKC........ DGTLFSCTNCSKIGFVCKISDRLTRSSFFKGYTKNLEOKL I DMELDRNRLMLELNR IKKEGF 56 WKRF SQACDRCRLKKIKC........ DGIKPSCSNCKKIGYHCSTSDKLTRRGFFRGYTEMLENEVIKLQ . . RLC.GMVDENGETW 39 SVRKAHACDRCRLKKIKC. . . . . . DGLKPNCSNCAKIDFPCKTSDKLSRRGLPKGYTELLEKEVURLT . . . . . . . . . . 17 DSRIADACDRCRSKKIRC ......... DGIRPCCTQCANVGFECKT SDKLSRRAFFRGYTESLEERVRTLEAEVRELK-SLLDEKDEKI 63 IYRIAQACDRCRSKKTRC......... DGKRPQCSQCAAVGFECRISDKLLRKAYPKGYTESLEERVRELEAENKRLL. ALCDIKEQO 176 SYRVAQACDRCRAKKIRC ........ DGKRPQCTQCAAVGFECKI SDKLSRRAFPRGYTETLEERVRELEAENRRLV.ALCDLKEEQL 29 IER TIRVSVACDRCRKKKI KCFYEEGEEVDFNGRTQCANCKAVGLDCIF TDKLARKAFPRGYTESLEERURELEFENKKLH. KLLKMRAENP

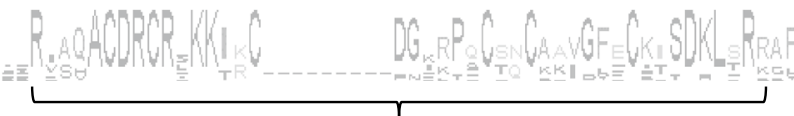

$\mathrm{Zn}(\mathrm{II})_{2} \mathrm{Cys}_{6}$ binuclear cluster DNA binding domain

Leucine zipper-like dimerization domain

B

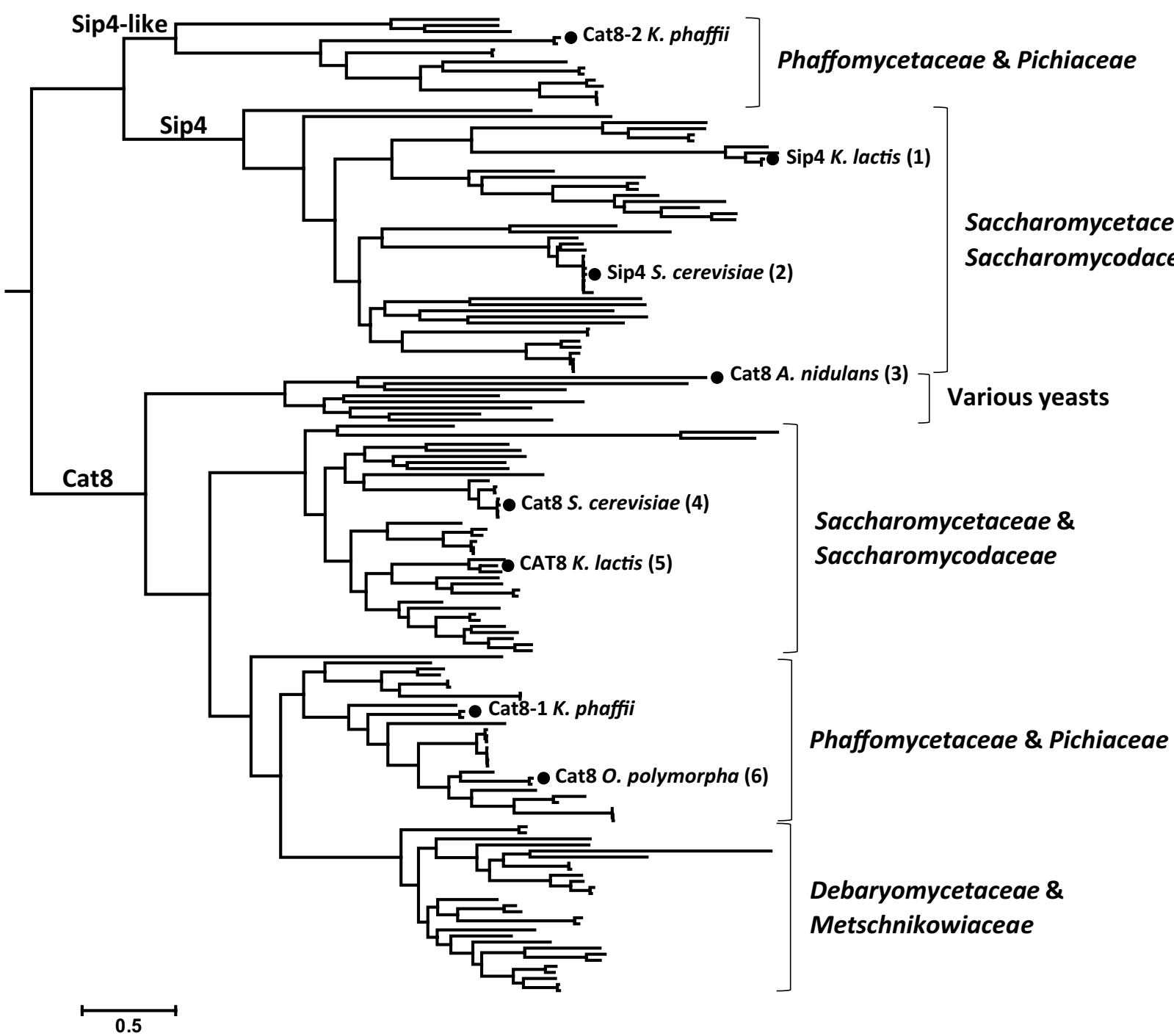

Fig. 1 Phylogenetic and sequence analysis of sequence homologs of Cat8 and Sip4. A Multiple sequence alignment and sequence logo of the fungal $\mathrm{Zn}(\mathrm{II})_{2} \mathrm{Cys}_{6}$ DNA binding domain of the 6 functionally characterized Cat8 and Sip4 as well as K. phaffii Cat8-1 and Cat8-2. Identical residues are shown in grey boxes and the cysteine residues involved in forming the DNA binding domain are marked with a star. B Phylogenetic tree based on 161 full length amino acid sequences of Cat8 and Sip4 homologs in Saccharomycetes (Yeast; taxid:4891). Full circles $(\bullet)$ represent the positions of the 6 functionally characterized Cat8 and Sip4 as well as $K$. phaffii Cat8-1 and Cat8-2 protein sequences that were used for BLAST search: 1. Mehlgarten et al. 2015, 2. Lesage et al. 1996, 3. Todd et al. 1997, 4. Hedges et al. 1995, 5. Georis et al. 2000, 6. Ruchala et al. 2017 
on ethanol $\left(0.20 \mathrm{~h}^{-1}\right.$ for cat $8-1 \Delta$ and $0.12 \mathrm{~h}^{-1}$ for cat $8-2 \Delta$ against $0.25 \mathrm{~h}^{-1}$ for the wild-type), and they reached a lower $\mathrm{OD}_{600}$ at the end of the culture (Fig. 2D). Furthermore, a cat8-1 $\Delta$ cat8-2 $\Delta$ double knock-out was generated and it was shown to be completely unable to grow on ethanol (Fig. 2D). When a single copy of the CAT8-1 or the CAT8-2 genes under the control of their native promoters was introduced in the cat $8-1 \Delta$ or cat $8-2 \Delta$ mutant strains, respectively, the growth was again similar to that of the wild-type, showing that the phenotype observed on ethanol is caused solely by the lack of these two genes (Supplementary Figure S1). In contrast, complementation of cat8-2A or the double knockout cat8- $1 \Delta$ cat8-2A with $S$. cerevisiae SIP4 (under control of the CAT8-2 promoter) did not rescue growth on ethanol (not shown), indicating that $\mathrm{ScSip} 4$ was neither able to complement Cat8-1 nor Cat8-2 under the analyzed conditions. Therefore, it was concluded that Cat8-1 and Cat8-2 are two essential transcription factors for the growth of $K$. phaffii on ethanol.

\section{Deletion of CAT8-1, but not of CAT8-2, impairs growth of $K$. phaffii on acetate}

Growth of the knock-out strains cat8- $1 \Delta$ and cat $8-2 \Delta$, and the cat8-1 $1 \Delta$ cat $8-2 \Delta$ double knock-out was also assessed on acetate, another $\mathrm{C} 2$ carbon source. As it has been reported that $K$. phaffii deleted for another TF, Mxr1, only showed impaired growth on complex medium, but not YNB (Sahu and Rangarajan 2016), we cultivated the strains on both YP-A (Fig. 2E) and YNB-A (Fig. 2F), using two different acetate concentrations ( 1 and $2 \%$ ) each. As can be seen in Fig. $2 \mathrm{~F}$, cat $8-1 \Delta$ and the double knock-out cat8- $1 \Delta$ cat $8-2 \Delta$ show impaired growth on acetate, which is more manifested on $2 \%$ acetate in YNB than in the other tested conditions. In YNB-A, growth rates were reduced to $77 \%$ in the cat8-1 $1 \Delta$ mutant and to $70 \%$ in the double knock-out on both acetate concentrations. A slight delay in growth was also observed for cat $8-2 \Delta$.

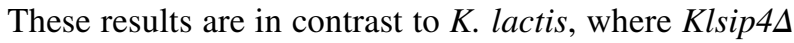
and the double knock-out Klsip $4 \Delta$ cat $8 \Delta$ could not grow on acetate at all, and Klcat $8 \Delta$ was growth impaired (Mehlgarten et al. 2015).

\section{CAT8-1 and CAT8-2 expression levels are higher on ethanol and methanol than on glucose and glycerol}

As a next step, the transcript levels of CAT8-1 and CAT8-2 in the wild-type induced on different carbon sources were determined.

Transcript levels of CAT8- 1 in $K$. phaffi $i$ wild-type were two- to fourfold increased on ethanol and methanol compared to glucose and glycerol (Fig. 3A) and transcript levels of CAT8-2 in K. phaffii wild-type were higher on glycerol, ethanol, and methanol than on glucose, with more than 500fold induction on the two alcohols (Fig. 3A). Generally, the expression of CAT8-1 remained quite low: around 4 and $6 \%$ compared to the actin gene (ACT1) expression on ethanol and methanol, respectively (Fig. 3B). Expression of CAT82 was much higher: $C A T 8-2$ reached around $60 \%$ of $A C T 1$ expression on ethanol and methanol (Fig. 3B), which is more than 10-times higher than the expression levels reached by CAT8-1.

Regarding the $C A T 8$ overexpression strains, there was around 15-fold up-regulation of CAT8-1 in the CAT8-1 overexpression strain on ethanol, whereas up-regulation was 150-fold in the same strain on methanol compared to the wild-type (Fig. 3C). For CAT8-2, we observed 2.34-fold up-regulation in the CAT8-2 overexpression strain on ethanol and a 40-fold up-regulation on methanol (Fig. 3C). It can therefore be concluded that the overexpression of CAT8-1 and CAT8-2 with $\mathrm{P}_{T H I 11}$ is stronger on methanol than ethanol. No effect of CAT8-1 overexpression on CAT8-2 mRNA levels or vice versa were observed.

\section{Cat8-1 and Cat8-2 are involved in regulating the expression of genes important for ethanol assimilation}

To identify potential target genes of Cat8-1 and Cat8-2, mRNA levels of 13 genes were analyzed in the overexpression and knock-out mutants of CAT8-1 and CAT8-2 as well as in the wild-type induced on different carbon sources. The investigated genes were selected because they were either important for the growth of yeasts on gluconeogenic carbon sources and reported to be under the control of Cat8 and/ or Sip4 in S. cerevisiae and K. lactis (ACS1, ADH2, ALD4, $C R C 1, F B P 1, I C L 1, M L S 1, P C K 1$, and YAT2) or involved $K$. phaffii methanol metabolism (AOX1, DAS1, PEX5, MXR1).

The samples for the transcript level analysis were obtained by growing the mutant strains and the wild-type in liquid cultures on minimal medium with limiting glucose to avoid glucose repression in the pre-culture and then shifting the cultures to $2 \%$ glucose, $2 \%$ glycerol, $1 \%$ methanol or $2 \%$ ethanol for $5 \mathrm{~h}$ to have an induction on either glucose, glycerol, methanol, and ethanol, respectively. Cells were then harvested and RNA was extracted for qRT-PCR (Fig. 4A).

The promoters of the genes selected for the transcript level analysis were screened in silico by MatInspector (Genomatix, Germany) for the presence of the CSRE motif representing the binding site of Cat8 and Sip4. All of the promoters of the selected genes were shown to possess at least one predicted CSRE motif, most of them having more than two of these motifs. In particular, the $C R C 1$ and the $A O X 1$ promoter possesses one CSRE, while the ICL1 and PEX5 promoters have two CSREs, the MLSI, MXRl, and 
A

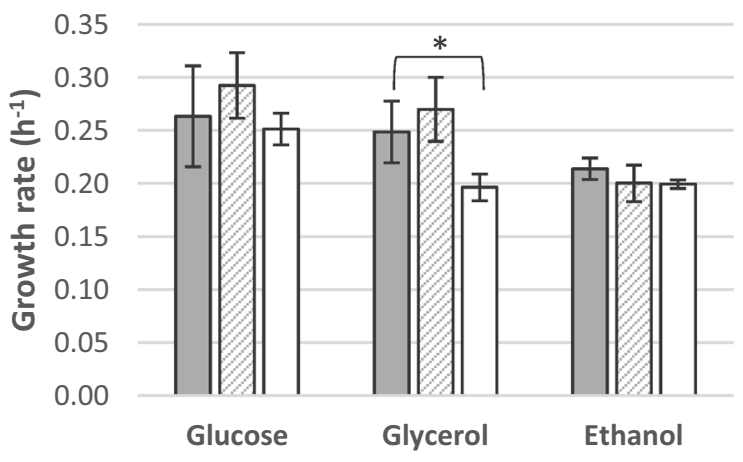

$\square W T \square C A T 8-1 \_O E \quad \square C A T 8-2 \_O E$

C

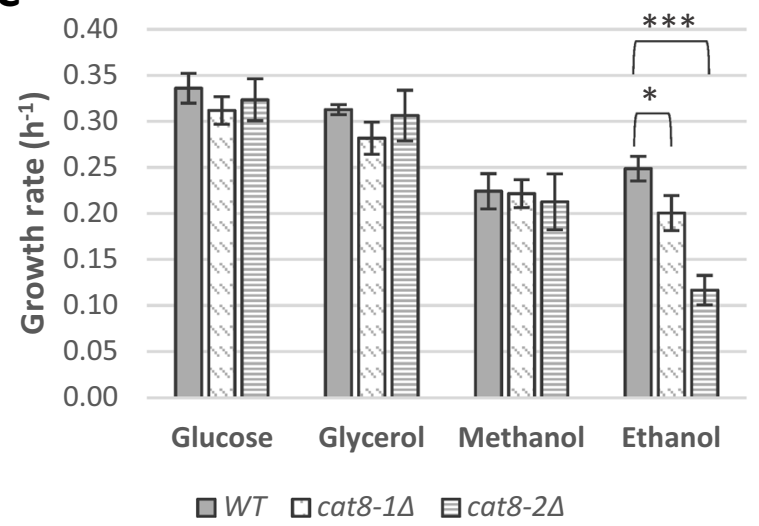

E

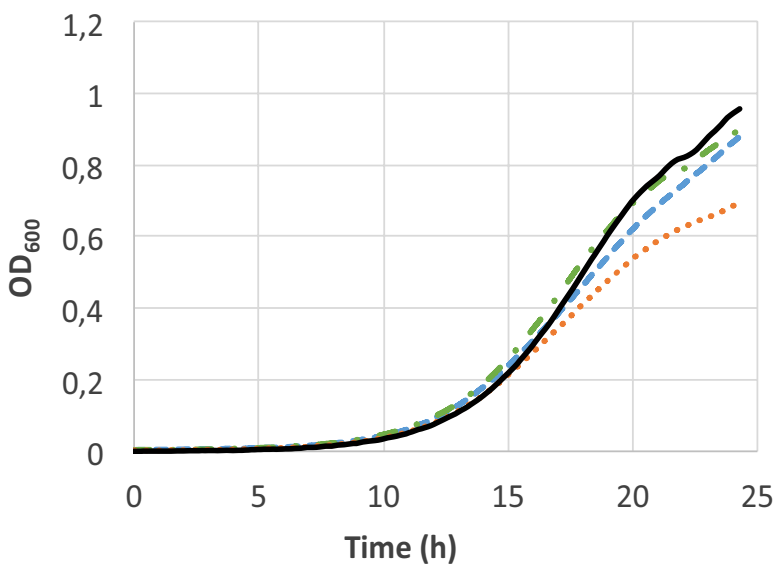

$-\cdot \operatorname{cat} 8-1 \Delta-\cdot \operatorname{cat} 8-2 \Delta$
B

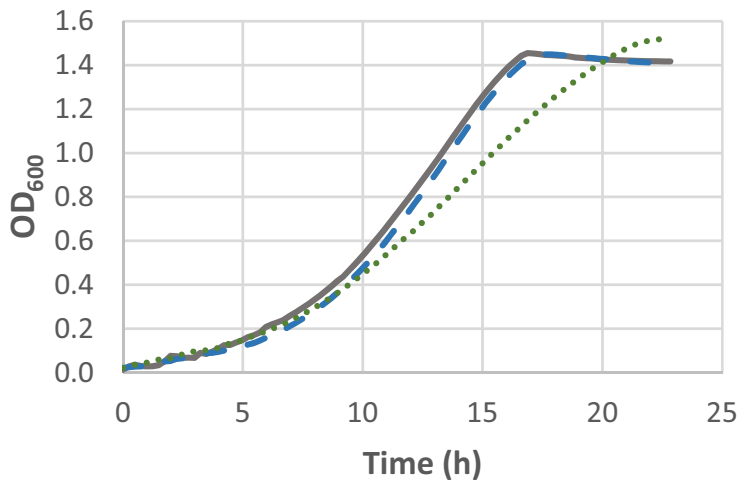

WT - - CAT8-1_OE

CAT8-2_OE

D

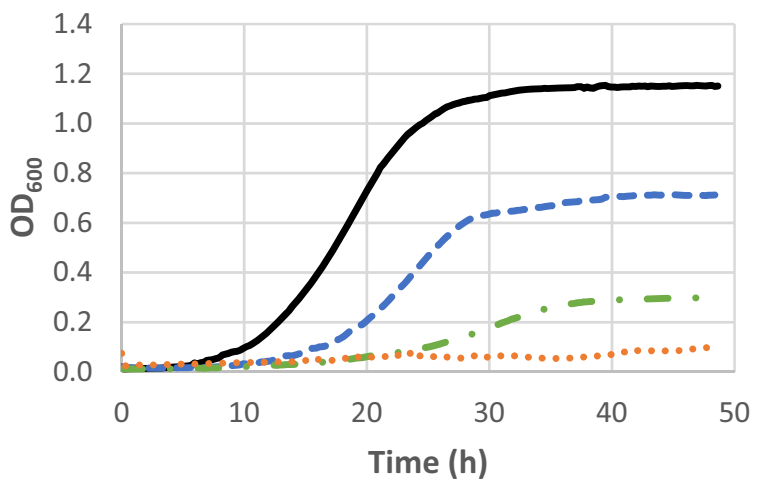

$\longrightarrow W T \quad \cdots-\infty \operatorname{cat} 8-1 \Delta$

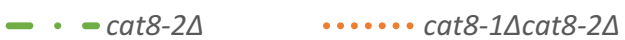

$\mathbf{F}$

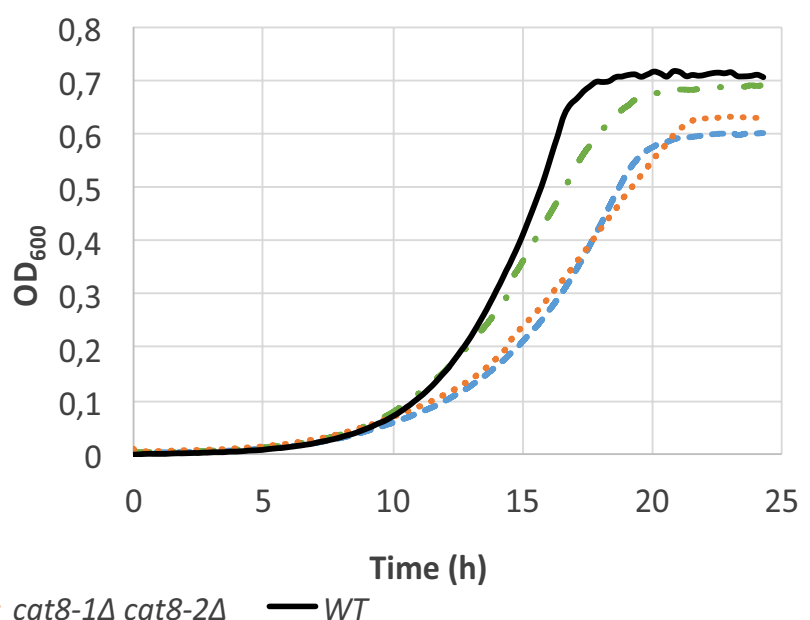

$A L D 4$ promoter three, the YAT2 and DAS1 promoters four, the $A D H 2$ and $F B P 1$ promoters five and finally the $P C K 1$ promoter eight predicted CSRE sites.
On glucose and glycerol, no significant difference in transcript levels of the selected genes was observed in the different Cat8 mutant strains compared to the wild-type (data not shown). 
4Fig. 2 Influence of CAT8-1 and CAT8-2 overexpression and deletion on carbon source utilization. A Growth rates of the CAT8-1 and CAT8-2 overexpression mutants and the $K$. phaffii wild-type (WT) on YNB without thiamine with $2 \%$ glucose, $2 \%$ glycerol and $1 \%$ ethanol. B Growth curves of the CAT8-1 and CAT8-2 overexpression mutants and the WT on YNB without thiamine with $2 \%$ glycerol. C Growth rates of cat $8-1 \Delta$, cat $8-2 \Delta$ and the WT on $2 \%$ glucose, $2 \%$ glycerol, $1 \%$ methanol and $1 \%$ ethanol. D Growth curves of cat $8-1 \Delta$, cat $8-2 \Delta$, the double knock-out cat8-1s cat $8-2 \Delta$ and the WT on $1 \%$ ethanol. E Growth curves of cat $8-1 \Delta$, cat $8-2 \Delta$, the double knock-out cat $8-1 \Delta$ cat $8-2 \Delta$ and the WT on $\mathrm{YP}+2 \%$ acetate. F Growth curves of cat $8-1 \Delta$, cat $8-2 \Delta$, the double knock-out cat $8-1 \Delta$ cat $8-2 \Delta$ and the WT on $\mathrm{YNB}+2 \%$ acetate. Error bars represent the standard deviations of three to four independent biological samples each measured in technical triplicates. The statistically significant differences compared to the WT are indicated with asterisks (Student's $t$ test; $* p<0.05$, $* * p<0.01, * * * p<0.001)$

On ethanol, the expression of the two gluconeogenic genes FBP1 and PCK1 (PP7435_Chr3-0309 and PP7435_ Chr1-1542 encoding fructose-1,6-bisphosphatase and phosphoenolpyruvate carboxykinase, respectively) were similar to that of the wild-type in the different mutant strains, showing that Cat8-1 and Cat8-2 are not involved in the regu-

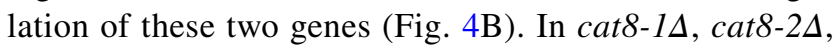
and in the cat8-1 $\Delta$ cat $8-2 \Delta$ double knock-out mutant, the transcript levels of three genes involved in ethanol assimilation, ADH2 (PP7435_Chr2-0821, encoding alcohol dehydrogenase II), ACS1 (PP7435_Chr2-0505, encoding acetylcoenzyme A synthetase I) and ALD4 (PP7435_Chr2-0787, encoding aldehyde dehydrogenase IV) were all decreased (Fig. 4B), showing that both Cat8-1 and Cat8-2 are involved in the regulation of the expression of these three genes. The expression of two genes from the glyoxylate shunt, ICLI (PP7435_Chr1-1123, encoding isocitrate lyase) and MLS1 (PP7435_Chr4-0820, encoding malate synthase), was decreased in cat8- $1 \Delta$ and in the cat8-1 $1 \Delta$ cat $8-2 \Delta$ double knock-out mutant but not in cat $8-2 \Delta$ when compared to the wild-type. Therefore, only Cat8-1 seems to be necessary for the activation of these genes from the glyoxylate shunt in $K$. phaffii. For two genes from the carnitine shuttle, YAT2 (PP7435_Chr3-0432, encoding cytosolic carnitine acetyl transferase) and CRC1 (PP7435_Chr2-0377, encoding carnitine carrier I), the expression was decreased in cat8-2 $\Delta$ and in the cat8-1 1 cat $8-2 \Delta$ double knock-out mutant but not in cat $8-1 \Delta$ when compared to the wild-type. Therefore, Cat8-2 seems to be necessary for the activation of these genes from the carnitine shuttle. Additionally, the expression of three genes important for methanol assimilation, $A O X 1$ (PP7435_Chr4-0130, encoding alcohol oxidase I), DAS1 (PP7435_Chr3-0352, encoding dihydroxyacetone synthase I) and PEX5 (PP7435_Chr2-0195, encoding a peroxisomal membrane receptor) was assessed in the TF mutant strains on ethanol, but no specific regulation pattern was observed (Supplementary figure S2): due to the very weak expression of these genes on ethanol, the small differences observed in expression were not considered significant. Additionally the expression levels of MXR1 (PP7435_Chr4-0490) were investigated. $M X R l$ expression on ethanol was approximately $60 \%$ of the expression on methanol, and was found to be approximately two-fold higher in all cat 8 deletion strains on ethanol (Supplementary figure S2).

Similar to what we observed on ethanol, on methanol we also found the necessity of Cat8-1 for the activation of ICLI and $M L S 1$, the two genes of the glyoxylate shunt, and the dependence of ACS1 and ALD4 on both TFs (Fig. 4C, D). No impact of the knock-outs on the other analyzed genes was observed after induction by methanol. In addition, we observed an increase in transcript levels for all the analyzed genes in the CAT8-2 overexpression mutant, but except for $A C S 1$ and $A L D 4$ there was no significant difference in expression seen in cat8-2A (Fig. 4C). ICL1 and MLS1 expression is also increased in the $C A T 8-1$ overexpression strain, albeit to a lower level than reached by CAT8-2 overexpression (Fig. 4C). Also on methanol, there was no impact of any of the Cat8 mutants on the methanol utilization genes (Supplementary figure S2).

\section{The CAT8-2 gene expression is autoregulated by the Cat8-2 protein}

In $K$. lactis and S. cerevisiae, the Cat8 protein regulates the expression of Sip4, and Sip4 autoregulates the activity of its own promoter (Mehlgarten et al. 2015). In both species, Cat 8 transcription factor binding sites (TFBS) are predicted in the SIP4 promoter regions (Supplementary Figure S3). In order to analyze a possible regulation of the CAT8- 1 and CAT8-2 genes by the Cat8-1 and Cat8-2 proteins in K. phaf$f i$, eGFP reporter strains were generated. The eGFP gene was expressed under the control of the CAT8- 1 and CAT8-2 promoters, respectively. These constructs were transformed into the different knock-out mutants (cat8-1 $1 \Delta$, cat $8-2 \Delta$ and cat $8-1 \Delta$ cat $8-2 \Delta$ ) and the wild-type. The obtained strains were cultivated on a minimal medium with limiting glucose and then shifted to either $2 \%$ glucose, $2 \%$ glycerol, $1 \%$ methanol or $2 \%$ ethanol for induction. After $5 \mathrm{~h}$ induction, the eGFP fluorescence was measured by flow cytometry (Fig. 5).

In all strains and on all the carbon sources, the eGFP fluorescence was lower when the eGFP gene was under the control of the CAT8-1 promoter $\mathrm{P}_{C A T 8-1}$ compared to when the eGFP gene was under the control of the CAT8-2 promoter $\mathrm{P}_{\text {CAT8-2 }}$ (compare axes in Fig. 5A, B), in accordance with the differences in transcript levels measured by qRTPCR (Fig. 3B). With the CAT8-1 promoter, no significant difference was observed in the fluorescence levels of the different strains on the different carbon sources (Fig. 5A). Thus, the expression of CAT8-1 does not seem to be affected by neither Cat8-1 nor Cat8-2. With the CAT8-2 promoter in the wild-type, the eGFP fluorescence was lower on glucose 
A
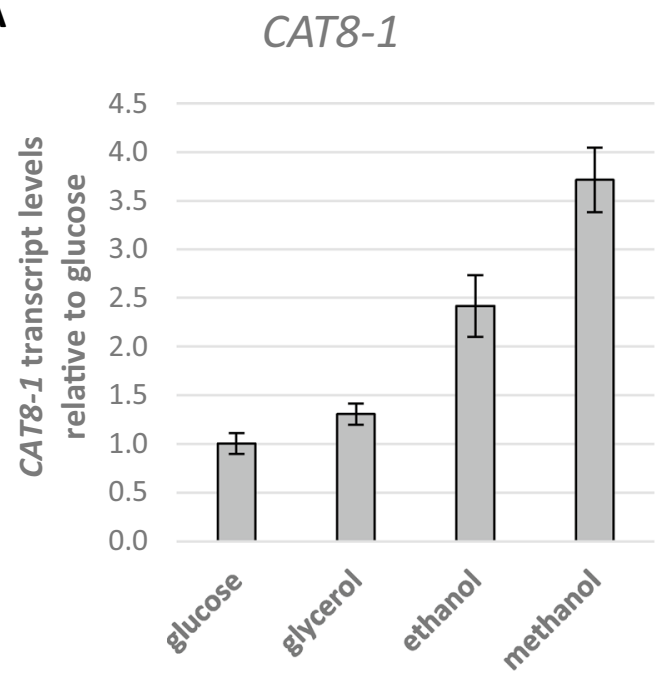

B

CAT8-1

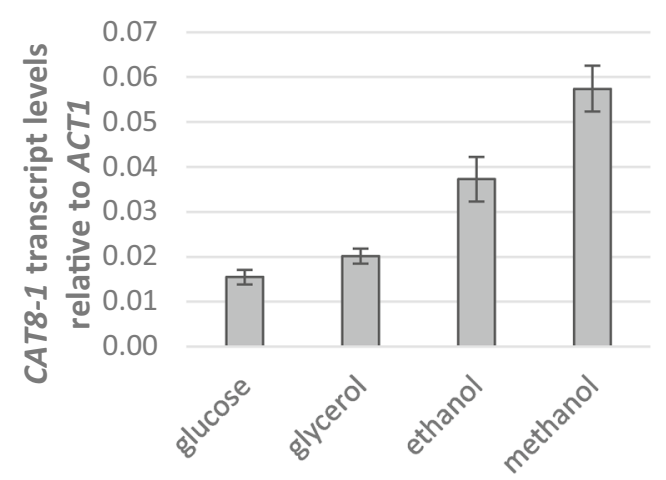

C

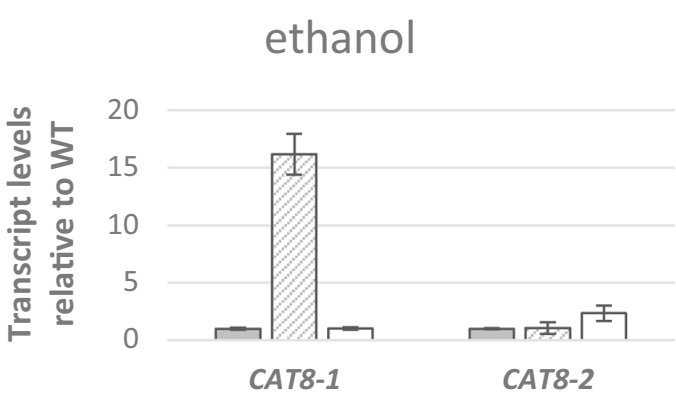

口WT $\square$ CAT8-1_OE $\square$ CAT8-2_OE

Fig. 3 Influence of the carbon source on CAT8-1 and CAT8-2 gene expression. A Transcript levels of CAT8-1 and CAT8-2 in the K. phaffii wild-type CBS7435 induced on different carbon sources. Gene expression levels were normalized to the reference gene ACT1 and quantified relative to the levels on glucose (set to 1.0) for each carbon source. B Expression strength of CAT8-1 and CAT8-2 in the K. phaffii wild-type CBS7435 on glucose, glycerol, ethanol and methanol relative to the $A C T 1$ gene. Error bars represent the standard deviations
CAT8-2
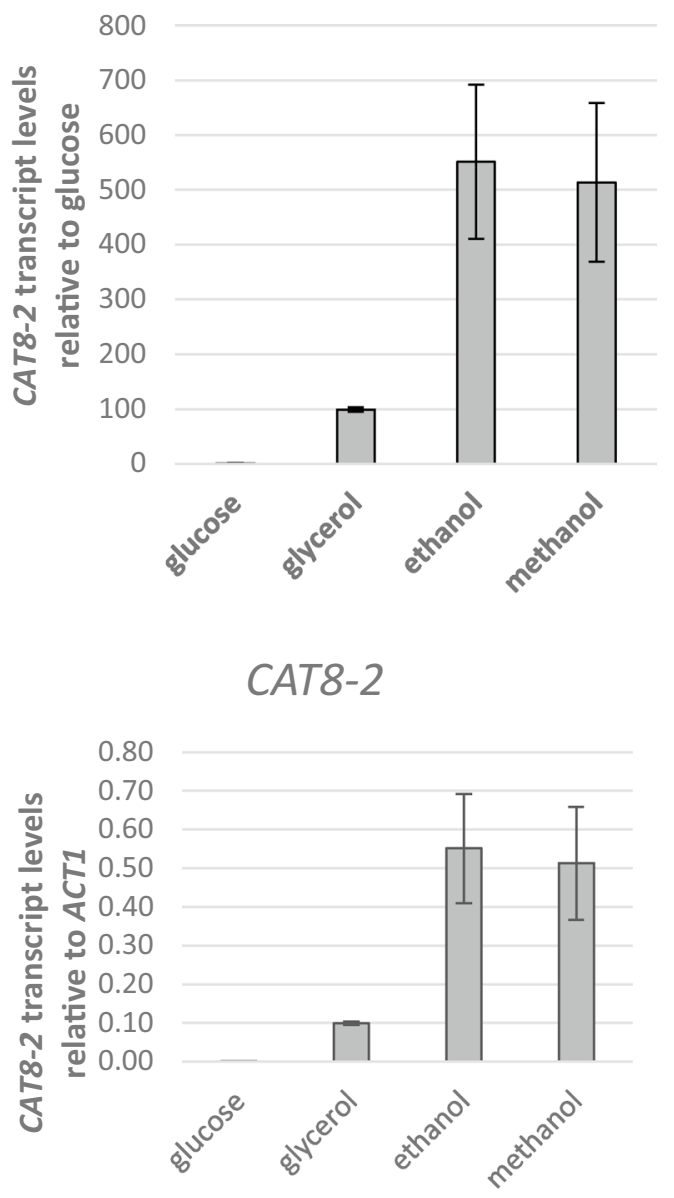

methanol

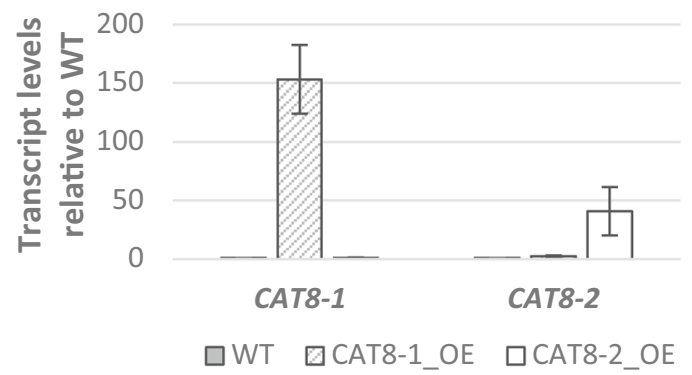

obtained with two biological samples each measured in technical triplicates in two independent experiments. C mRNA levels of CAT8-1 and $C A T 8-2$ in overexpression mutants and the $K$. phaffi $i$ wild-type on ethanol and methanol. Gene expression levels were normalized to the reference gene $A C T 1$ and quantified relative to wild type (WT) levels (set to 1.0) for each carbon source. Error bars represent the standard deviations of two biological samples each measured in technical triplicates in two independent experiments 
A

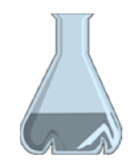

Pre-culture YPD

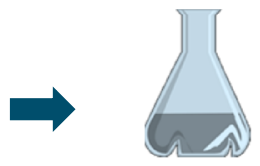

$\mathrm{OD}_{600} \approx 4$

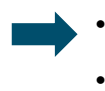

- Wash cells $\times 2$ in $\mathrm{H}_{2} \mathrm{O}$

Main culture ASM limiting glucose

- $\quad$ Add ASM + carbon source

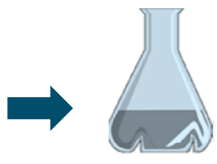

Induction

5 hours

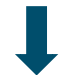

Samples for qRT-PCR

B

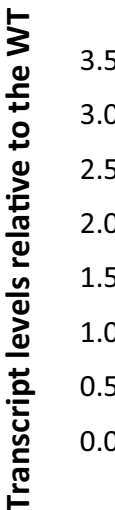

.5
3.0
2.5
2.0
1.5
1.0
0.5
0.0

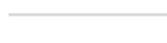

ethanol

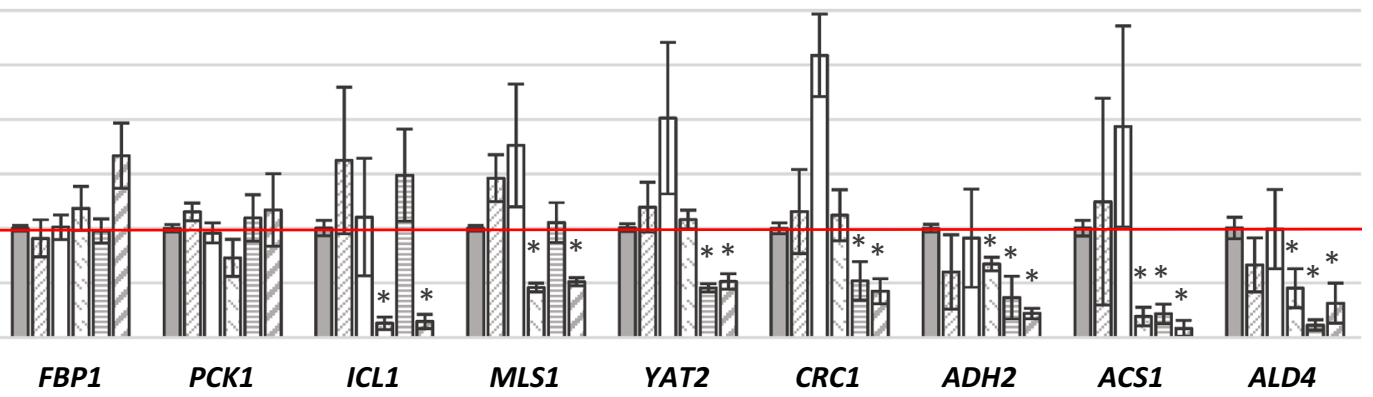

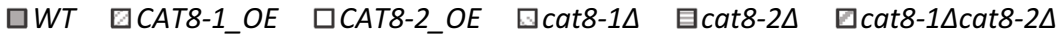

C

methanol

党

100

80

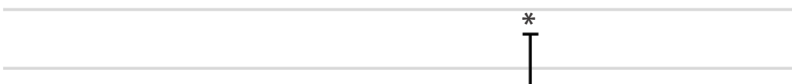

$\Gamma^{*}$

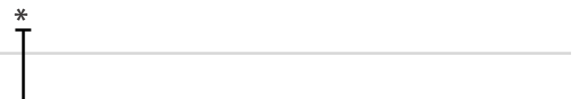

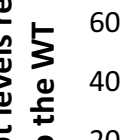

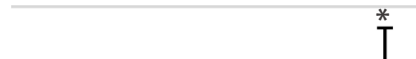

0

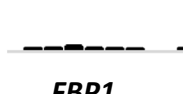

两

PCK1

ICL1 MLS1

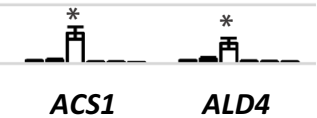

D

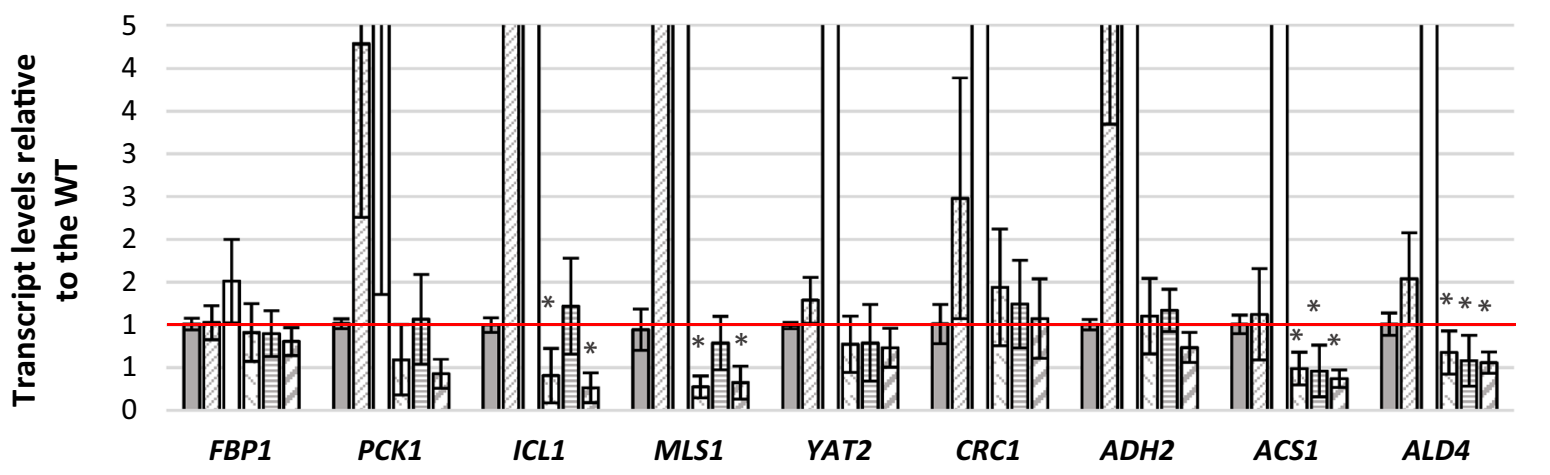

口WT $\square$ CAT8-1_OE पCAT8-2 OE

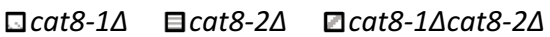

Fig. 4 Influence of CAT8-1 and CAT8-2 overexpression and knockout on transcript levels of selected genes in K. phaffii induced on ethanol and methanol. A Scheme of the sampling for the transcript level analysis. Transcript levels of selected genes in CAT8-1_OE, CAT8-

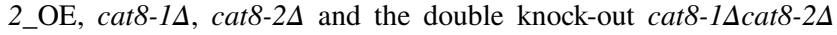
induced on $2 \%$ ethanol $(\mathbf{B})$ or on $1 \%$ methanol $(\mathbf{C}, \mathbf{D})$, determined by qRT-PCR. Gene expression levels were normalized to the refer- ence gene $A C T 1$ and quantified relative to wild-type (WT) levels (WT set to 1.0, red line). D Shows a zoom into C. Error bars represent the standard deviations of two independent biological samples each measured in technical triplicates in two independent experiments. The statistically significant differences compared to the WT are indicated with asterisks (Student's $t$ test; $\nabla p<0.01, * p<0.001$ ) 
A

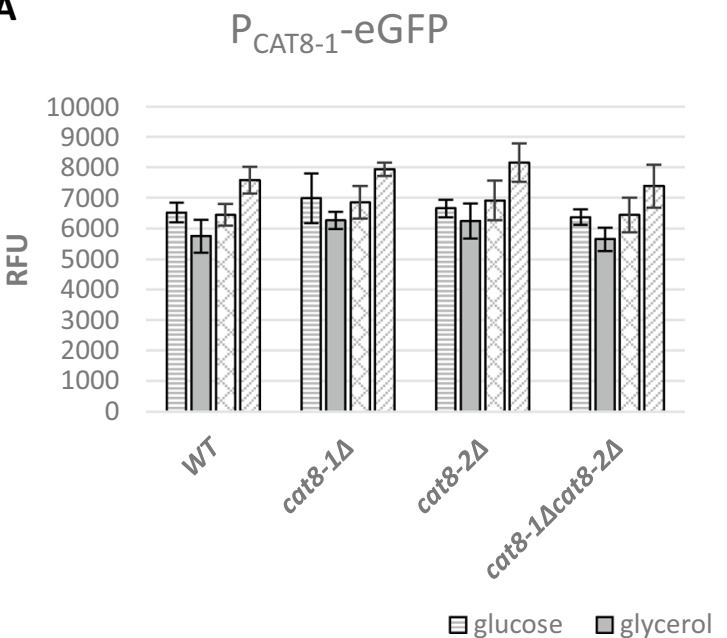

Fig. 5 Influence of CAT8-1 and CAT8-2 deletions on CAT8-1 and CAT8-2 promoter activity. Relative fluorescence units (RFU) obtained for A $\mathrm{P}_{\text {CAT8-1 }}$-GFP and B $\mathrm{P}_{\text {CAT8-2- }}$ GFP (1000 bps upstream of the respective $C A T 8$ gene fused to eGFP as reporter gene) in wild-

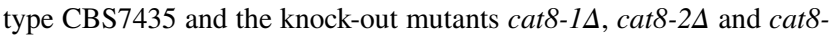
$1 \Delta$ cat $8-2 \Delta$. All strains carrying the eGFP reporter construct as well

and glycerol than on ethanol and methanol (Fig. 5B), which fits to the results obtained for the transcript level analysis of the CAT8-2 gene on these four carbon sources (Fig. 3A). In the cat8-1 $1 \Delta$ mutant, the eGFP levels under control of $\mathrm{P}_{\text {CAT8-2 }}$ were similar to the ones measured in the wild-type on each of the four carbon sources (Fig. 5B). However, in the cat $8-2 \Delta$ and cat $8-1 \Delta$ cat $8-2 \Delta$ mutants, the eGFP levels under control of $\mathrm{P}_{C A T 8-2}$ were higher than the ones of the wildtype on glucose, glycerol, ethanol, and methanol (Fig. 5B). Therefore, it seems that Cat8-1 is not regulating the expression of CAT8-2, but that the Cat8-2 protein is repressing/ autoregulating its own promoter on all tested carbon sources.

\section{The Mig1-2 transcription factor is involved in regulating CAT8-2 expression}

The transcription of $C A T 8$ was described to be regulated by the transcription factor Mig1 in S. cerevisiae (Carlson 1999; Schüller 2003). Correspondingly, there are 4 predicted Mig1 transcription factor binding sites upstream of $S$. cerevisiae CAT8 (Supplementary Figure S3). K. phaffii possesses two gene homologs of this transcription factor, termed MIG1-1 (PP7435_Chr4-0661) and MIG1-2 (PP7435_Chr1-1325). To investigate whether Mig1-1 and Mig1-2 are involved in the transcriptional regulation of CAT8-1 and CAT8-2 in K. phaffii, their transcript levels were analysed in knock-out and overexpression strains of the two Mig transcription factors (Ata et al. 2017). The Mig1-1 and Mig1-2 mutant strains were first cultivated on minimal medium with limiting glucose until $\mathrm{OD}_{600}$ reached 4 , and then transferred to $2 \%$ glucose, $2 \%$ glycerol,
B

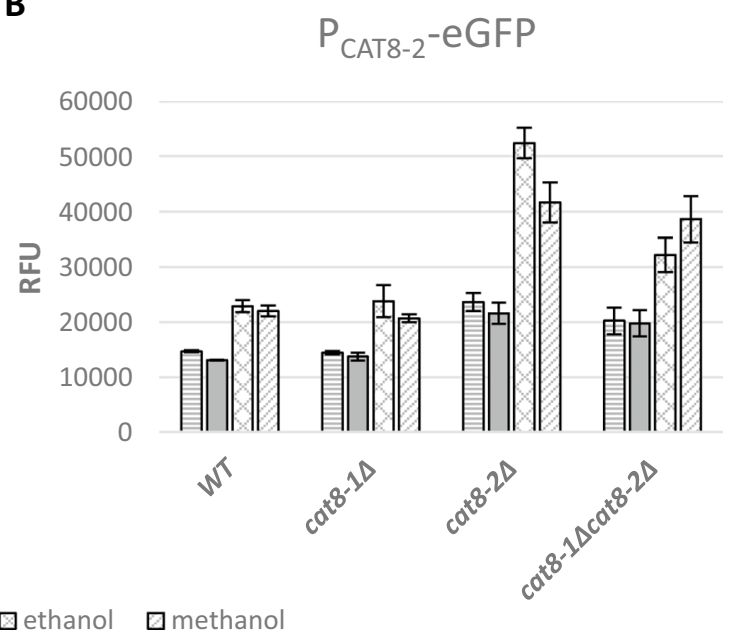

as a non-transformed negative control were grown in biological triplicates on minimal media with limiting glucose and then shifted to glucose, glycerol, ethanol or methanol for $5 \mathrm{~h}$. The eGFP fluorescence was measured by flow cytometry. Mean values and standard deviation for the three biological replicates are presented

$1 \%$ methanol or $2 \%$ ethanol for induction. After $5 \mathrm{~h}$ induction, samples were processed and the transcript levels of CAT8-1 and $C A T 8-2$ were measured in the different strains induced on the different carbon sources (Fig. 6).

The CAT8-1 transcript levels were similar to that of the wild-type in all mutant strains and on all carbon sources (Fig. 6). Therefore CAT8-1 does not seem to be regulated by neither Mig1-1 nor Mig1-2 in the conditions studied. The expression of CAT8-2 on the other hand is around 30-fold higher in the mig 1-2 $2 \Delta$ mutant than in the wild-type on glucose (Fig. 6A) and clearly down-regulated on glycerol in the MIG1-2_OE mutant (Fig. 6B). Indeed, there are 5 predicted Mig1-TFBS upstream of $C A T 8-2$, but only one very distant Mig1-TFBS in the $1000 \mathrm{bps}$ upstream of CAT8-1 (Supplementary figure S3). Finally, the expression of CAT8-2 is also lower than in the wild-type on ethanol in the MIGI-2 $O E$ mutant (Fig. 6C) and not affected at all on methanol (Fig. 6D). CAT8-2, therefore, seems to be repressed in the presence of Mig1-2 on glucose and glycerol, but not on ethanol unless MIG1-2 is artificially overexpressed. Transcript levels of genes regulated by Cat8-1 and Cat $8-2$ were also measured in the Mig1-1 and Mig1-2 mutant strains, but no significant difference was observed compared to the wildtype (Supplementary figure S4).

\section{Investigation of Cat8-1 and Cat8-2 post-translational activation}

Cat8 and Sip4 are both activated through phosphorylation in S. cerevisiae: Cat8 was shown to be phosphorylated by the 
A

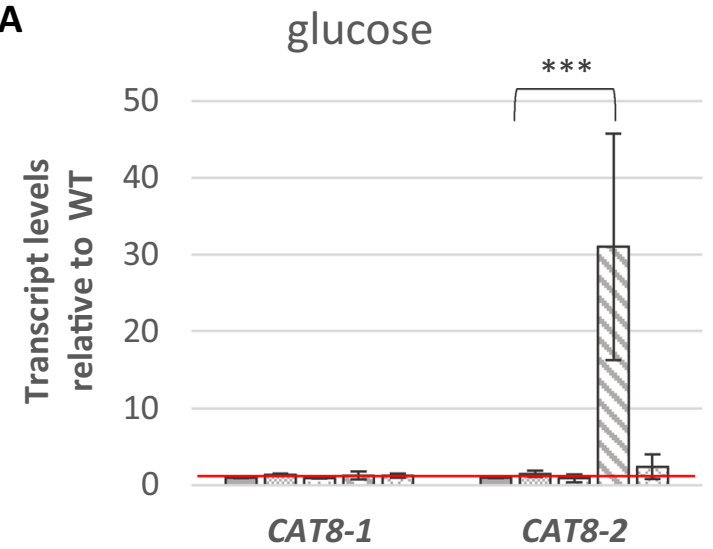

C

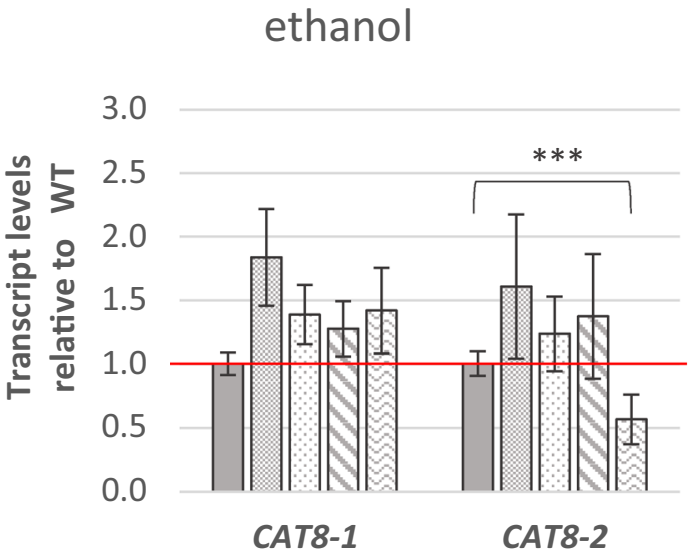

B

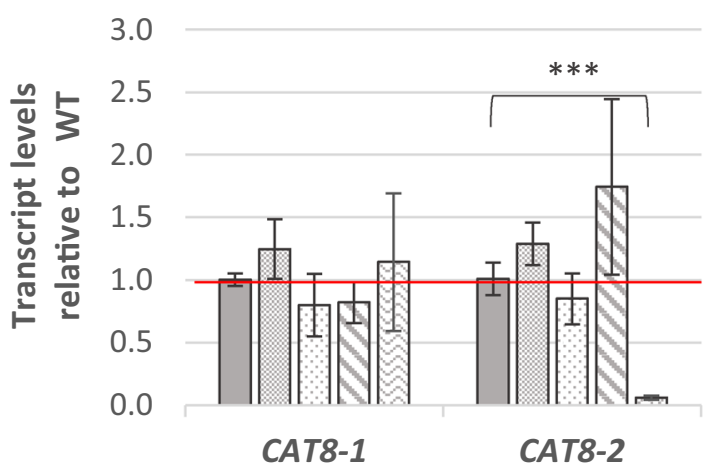

D

\section{methanol}

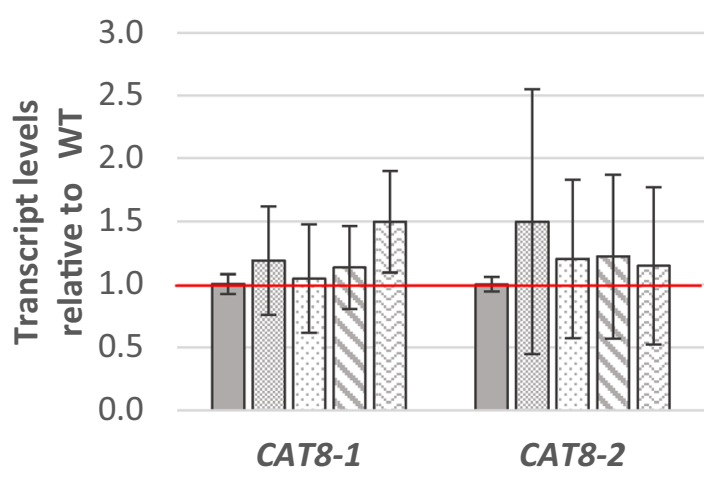

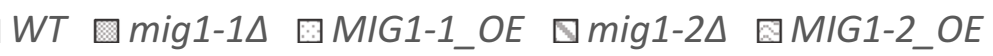

Fig. 6 Influence of MIG1-1 and MIG1-2 deletion and overexpression on CAT8-1 and CAT8-2 transcript levels. Transcript levels of CAT81 and CAT8-2 in mig1-1A, MIG1-1_OE, mig1-2L and MIG1-2_OE mutants induced on A glucose, $\mathbf{B}$ glycerol, $\mathbf{C}$ ethanol or $\mathbf{D}$ methanol relative to wild-type strain were determined by qRT-PCR. Gene expression levels were normalized to the reference gene ACTl and

yeast homolog of AMPK, the Snf1 kinase complex (Charbon et al. 2004; Randez-Gil et al. 1997), whereas Sip4 was shown to be phosphorylated by both the Snf1 kinase complex (Lesage et al. 1996; Vincent and Carlson 1999) and the Ssn3 kinase (Vincent et al. 2001). Thus, to assess the necessity of post-translational activation of Cat8-1 and/or Cat8-2 in K. phaffii, three kinases were selected for deletion: Snf1, Snf1-2 (both homologs of ScSnf1, encoded by PP7435_Chr2-0772 and PP7435_Chr1-0450, respectively), and Ssn3 (homolog of ScSsn3 encoded by PP7435_Chr11091). The generation of the SNF1-2 and SSN3 knock-out mutants was successful, but unfortunately, no transformants deleted for $S N F 1$ could be obtained.

The growth of the snfl-2A and $\operatorname{ssn} 3 \Delta$ deletion strains, as well as the $K$. phaffii wild-type, was assessed in liquid quantified relative to wild-type levels (WT set to 1.0, red line) for each carbon source. Error bars represent the standard deviations of with two independent biological samples each measured in technical triplicates in three independent experiments. The statistically significant differences compared to the WT are indicated with asterisks (Student's $t$ test; ***p<0.001)

cultures with YNB containing either $2 \%$ glucose, $2 \%$ glycerol, $1 \%$ ethanol or $1 \%$ methanol (Supplementary figure S5). No difference was observed between the specific

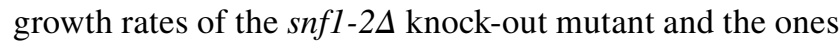
of the wild-type on the four-carbon sources tested. However, the specific growth rate of the $s s n 3 \Delta$ deletion strain is lower than that of the wild-type on glucose $\left(0.26 \mathrm{~h}^{-1}\right.$ for $\operatorname{ssn} 3 \Delta$ and $0.31 \mathrm{~h}^{-1}$ for the wild-type), but not on glycerol, ethanol nor methanol (Supplementary figure S5). These results suggest that the two kinases Snf1-2 and Ssn3 are not necessary for activation of Cat8-1 and Cat8-2 on ethanol. 


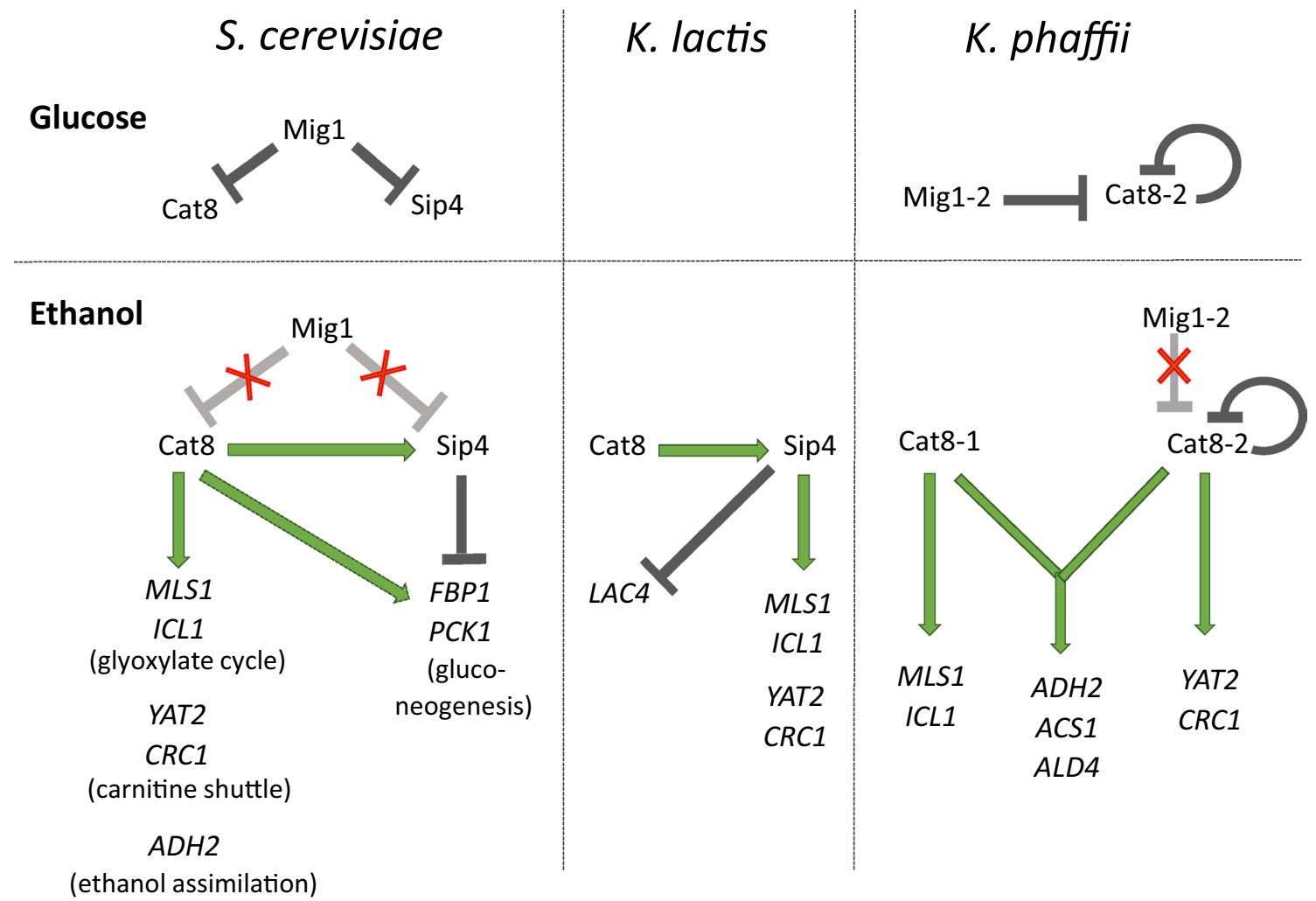

Fig. 7 Comparison of the Cat8-Sip4 regulatory networks in S. cerevisiae, $K$. lactis and $K$. phaffii. Schematic representation of the regulation by the transcription factors Cat 8 and Sip4 for selected genes.

\section{Discussion}

In this work, we studied the functions of two transcription factors named Cat8-1 and Cat8-2 in K. phaffii, which were identified as homologs of the transcription factor Cat8 from S. cerevisiae based on sequence similarity (Valli et al. 2016). These two TFs showed differential expression on methanol (Prielhofer et al. 2015) but were otherwise uncharacterized.

\section{K. phaffii Cat8-2 is a Sip4-like protein}

The Cat 8 and Sip4 homologous protein sequences used in the phylogenetic analysis showed a conserved DNA binding- and Leucine-zipper dimerization domain, but have a high variation for the rest of the sequences. Based on the inferred phylogenetic tree, the Cat8-1 protein sequence from $K$. phaffii was confirmed to be a Cat8 homolog, whereas Cat8-2 is more closely related to Sip4 protein homologs. While S. cerevisiae SIP4 was not able to complement the loss of $K$. phaffii Cat8-2, we identified some common features (see Figs. 4, 7) shared by Cat8-2 and Sip4 in the regulation of non-fermentable carbon sources
Arrows in green indicate transcriptional activation, whereas lines in dark grey indicate transcriptional repression. Elevated repression or derepression is indicated by light grey lines with red crosses

in $K$. phaffi, S. cerevisiae and $K$. lactis. Taken all our findings together, Cat8-2 can be considered a Sip4-like protein in K. phaffii.

\section{Role of Cat8-1 and Cat8-2 in the regulation of ethanol utilization}

When shifted to a gluconeogenic carbon source, yeast cells undergo a massive reprogramming of their gene expression, including genes involved in gluconeogenesis, the glyoxylate cycle, and tricarboxylic acid cycle. Ethanol is metabolized by an alcohol dehydrogenase (encoded by $A D H 2$ ) to acetaldehyde, which is subsequently converted into acetate by aldehyde dehydrogenase $(A L D)$. Acetate is then transformed into acetyl-Coenzyme A by acetyl-CoA synthetase $(A C S)$ in the cytoplasm. Then, acetyl-CoA must be transferred to the mitochondria for the production of energy (Schmalix and Bandlow 1993; Stemple et al. 1998). Two pathways exist to transport acetyl-CoA into the mitochondria: (1) acetyl-CoA is converted into glyoxylate cycle intermediates which are transported to the mitochondria (Palmieri et al. 1997) and (2) acetyl-CoA is converted into acetylcarnitine, which is transported into mitochondria via the carnitine shuttle. In addition to the carnitine shuttle and the glyoxylate cycle, 
gluconeogenesis is fundamental for the growth of non-fermentable carbon sources: it is essential for the production of glucose-6-phosphate, which is crucial for cell growth (Barnett and Entian 2005).

Both Cat8-1 and Cat8-2 were shown to be essential for the growth of $K$. phaffii on ethanol (Fig. 2), specifically, both transcription factors are required for the activation of $A D H 2, A L D 4$ and ACS1, three genes encoding enzymes important for assimilation of ethanol (Fig. 4A). But while Cat8-1 seems to be necessary for the activation of the glyoxylate cycle (by regulating $M L S 1$ and ICL1), Cat8-2 seems to be necessary for the activation of the carnitine shuttle (by regulating YAT2 and $C R C 1$ ). In contrast, the gluconeogenic genes $F B P 1$ and $P C K 1$ are not regulated by neither Cat8-1 nor Cat8-2 (Fig. 4). Furthermore, we could show that the CAT8-2 gene is clearly repressed in the presence of the Mig 1-2 transcription factor (a homolog of S. cerevisiae Mig1) on glucose and to a lesser extent also on glycerol (Fig. 6), and repressed/autoregulated by the Cat8-2 protein on glucose, glycerol, ethanol, and methanol (Fig. 5).

Altogether, we can observe three major differences in the regulation of genes for ethanol utilization by Cat8/Cat8-1 and Sip4/Cat8-2 in the three yeast species $S$. cerevisiae, $K$. lactis and K. phaffii (Fig. 7): (1) Cat8 is involved in the regulation of the gluconeogenesis in S. cerevisiae but neither in K. lactis nor in K. phaffii, (2) the expression of Sip4 is activated by Cat8 in S. cerevisiae and $K$. lactis but not in K. phaffii, and (3) the dissimilar importance of Cat8 and Sip4 in the different yeasts: although the same set of genes are targeted by Cat8/Sip4 and Cat8-1/Cat8-2 (except for the gluconeogenesis genes), the specific TF regulating the individual genes varies in the different species.

\section{Regulation of the gluconeogenesis}

In $S$. cerevisiae, Cat 8 and Sip4 are involved in the regulation of genes of the gluconeogenesis, therefore knocking out the genes encoding these two TFs results in a growth defect on glycerol (Hedges et al. 1995; Rahner et al. 1996). In $K$. phaffii as well as in K. lactis, when CAT8-1 and CAT8-2 (or CAT8 and SIP4) are knocked-out, there is no impairment of the growth on glycerol. In addition, transcript level analysis in $K$. phaffii showed that CAT8-1 and CAT8-2 deletions did not have a major influence on $F B P 1$ and $P C K 1$ expression on any of the tested carbon sources (Fig. 4 for ethanol and methanol, not shown for glucose and glycerol). Therefore, the regulation of gluconeogenesis seems to be achieved by a different regulatory network in $K$. phaffii compared to $S$. cerevisiae. One TF that could potentially play an important role in such a regulatory network is Rds2, which was shown to have partially overlapping functions with Cat8 in $S$. cerevisiae and to be a major regulator of gluconeogenesis. Specifically, Rds2 was shown to directly activate the expression of the gluconeogenic genes while repressing the negative regulators of this pathway, possibly through binding of the CSREs (Soontorngun et al. 2007). To our knowledge, the homolog of Rds2 in K. phaffii (PP7435_Chr2-1080) was not studied so far but could be involved in the regulation of gluconeogenesis in this yeast.

\section{Activation of SIP4 expression by CAT8}

In both $S$. cerevisiae and $K$. lactis, Cat8 has an important role in activating Sip4 expression on ethanol. Despite the fact that both CAT8-1 and CAT8-2 promoters carry three and two CSRE motifs, respectively (Supplementary File 2), this phenomenon is not observed in $K$. phaffii, suggesting that rewiring of the regulatory network of the carbon metabolism has happened somewhere during evolution.

Direct targets of CAT8 and SIP4 vary in the different yeast species In $S$. cerevisiae, Cat8 is the main activator for the growth of gluconeogenic carbon sources. In fact, the SIP4 deletion mutants do not exhibit any growth defect on any carbon source tested so far (Lesage et al. 1996), and the role of $S c \operatorname{Sip} 4$ is still unclear. $S c$ Cat8, on the other hand, was shown to activate the expression of genes from gluconeogenesis, the glyoxylate cycle, the ethanol assimilation pathway, and the carnitine shuttle. In K. lactis, Cat 8 activates the expression of Sip4 but does not seem to directly activate the expression of genes important for the growth of gluconeogenic carbon sources. Instead, KlSip4 is important for the regulation of the glyoxylate cycle and the carnitine shuttle. In $K$. phaffii, some genes seem to be specifically regulated only in the presence of either Cat8-1 or Cat8-2, while others seem to be overlapping targets of these two TFs. Indeed, Cat8-1 seems to be necessary for the activation of the genes from the glyoxylate cycle on ethanol, acetate, and methanol, while Cat8-2 seems to be important for the activation of genes from the carnitine shuttle on ethanol. As only cat8-1 $1 \Delta$, but not cat $8-2 \Delta$, showed clearly reduced growth on acetate, it seems that on acetate the glyoxylate cycle is the major active pathway for acetyl-CoA shuttling. In addition, Cat8-1 and Cat8-2 are both involved in the regulation of $A D H 2$, $A L D 4$ and $A C S 1$, three genes important for the conversion of ethanol to acetyl-CoA (Fig. 4), although the contribution of each TF for the regulation of these three genes remains to be investigated.

\section{Cat8-1 and Cat8-2 possibly recognize different subsets of CSRE motifs}

It was shown in $S$. cerevisiae that mutant CSREs show differential activation by Cat8 and Sip4 (Roth et al. 2004). In addition, the purified DNA binding domain of the TF Rds2 was shown to bind to the CSREs of the PCKI and FBPI 
promoters (Soontorngun et al. 2007). It was hypothesized that these three TFs bind subsets of CSREs with diverging affinities, which would allow for the regulation of both distinct and common target genes. This phenomenon could also be present in $K$. phaffii: transcript-level analysis showed that on methanol in the CAT8-2 overexpression strain, there are increased transcript levels of YAT2 and $C R C 1$ as well as ICLI and MLS1, two genes mainly regulated by Cat8-1, whereas in the $C A T 8-1$ overexpression, there is an increase only in ICL1 and MLS1 transcript levels (Fig. 4). This suggests that Cat8-1 and Cat8-2 could each have specific binding sites, but that, when Cat8-2 is present in high amounts, like on methanol and when strongly overexpressed, Cat $8-2$ could also bind a motif otherwise specific for Cat8-1. However, further experiments such as chromatin immunoprecipitation (ChiP) assay need to be performed in the future to investigate the specific DNA binding sites of Cat8-1 and Cat8-2.

In S. cerevisiae, ADH2 (Walther and Schüller 2001) and ACS1 (Kratzer and Schüller 1997) were reported to be synergistically activated by Cat 8 and Adr 1 . The sole homolog of Adr1 in K. phaffii is termed Mxr1 (methanol expression regulator 1) due to its function as activator of methanol utilization genes such as AOX1 (Lin-Cereghino et al. 2006; Kranthi et al. 2010). While Mxr1 is essential for the utilization of methanol, mutants lacking Mxr1 are still able to grow on other carbon sources including ethanol, albeit with a prolonged generation time (Lin-Cereghino et al. 2006). Mxr1 has recently also been implicated with the regulation of ACSl of $K$. phaffi grown on acetate in complex YP-containing media (Sahu and Rangarajan 2016). Expression levels of MXR1 are approximately $40 \%$ lower on ethanol than on methanol, and there is some indication that Cat8-1 and Cat8-2 are involved in repressing MXR1 expression on ethanol (Supplementary Figure S2), however, we do not know if this is due to direct binding of the TFs to the CSRE present in the MXRI promoter region or not. Additionally, there seems to be some regulatory effect of the overexpression of one transcription factor on the expression of the other on methanol, but the increased $M X R 1$ levels were not reflected by higher levels of Mxr1 target genes such as AOX1, DAS1 or PEX5 (Supplementary Figure S2). This fits the recent observation that integration of at least one $S c$ Cat8-TFBS into the $A O X 1$ promoter was needed in order to make the promoter responsive to ethanol (Ergün et al. 2020). Vice versa, no effect of Mxr1 overexpression on $\mathrm{P}_{A D H 2}$-GFP (Ergün et al. 2019) was seen on ethanol. Future research should be directed towards the interrelation of Cat8-1 and Cat8-2 with the Adr1-homolog Mxr1 and other carbon-source responsive transcriptional regulators in $K$. phaffii.

\section{Mechanisms of activation of Cat8 family members}

In the presence of glucose, expression of CAT8 and SIP4 is inhibited by the TF Mig1 and the Ssn6/Tup1 corepressor complex in S. cerevisiae (Carlson 1999; Schüller 2003). K. phaffii possesses two homologs of ScMig1: Mig1-1 and Mig1-2. These two TFs were previously shown to be involved in the regulation of the methanol metabolism in K. phaffii: derepres-

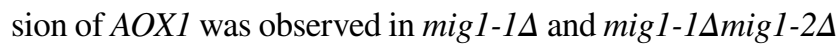
knock-out strains on glycerol (Wang et al. 2017), and the genes of the methanol metabolism, as well as the peroxisomes biogenesis pathway, were upregulated on glycerol in mig 11 $\Delta m i g 1-2 \Delta$ (Shi et al. 2018). Finally, Mig1-1 and Mig1-2 localized in the nucleus of cells grown on glucose or glycerol, but when K. phaffii cells were transferred to methanol, Mig1-1 and Mig1-2 predominantly localized to the cytoplasm (Wang et al. 2017).

In our experiments, we observed repression of CAT8-2 on glucose and glycerol by Mig1-2, but not on the two alcohols. No activation of the Cat8-2 target genes was observed in the Mig1-1 and Mig1-2 deletion strains, which can be explained by the involvement of further TFs in the regulation of these genes on glucose and/or glycerol.

We furthermore wanted to assess the necessity of posttranslational activation of Cat8-1 and/or Cat8-2, since Cat8 and Sip4 are both activated upon phosphorylation by the Snf1 and/or the Ssn3 kinases in S. cerevisiae (Charbon et al. 2004; Randez-Gil et al. 1997; Lesage et al. 1996; Vincent and Carlson, 1999; Vincent et al. 2001). The homolog of $S c \operatorname{Ssn} 3$ and the two putative homologs of $S c S n f 1$ present in K. phaffi, Snf1, and Snf1-2, were therefore selected for deletion. Unfortunately, the generation of the $S N F 1$ knock-out mutant was not successful. Since disruption of Snf1 was also not possible in the approach by Shen et al. (Shen et al. 2016), we think Snf1 to be essential in K. phaffii under the investigated conditions. Additionally, Snf1 was already reported to be essential in other non-Saccharomyces yeast species (Hedbacker and Carlson 2008).

No difference was seen in the growth of the snfl-2A and $\operatorname{ssn} 3 \Delta$ deletion strains compared to the wild-type on ethanol (Supplementary figure S4), hence these two kinases do not seem to play a major role in the regulation of ethanol utilization in $K$. phaffii, and are also probably not involved in the activation of Cat8-1 and Cat8-2 on this carbon source. Further investigations regarding the post-translational modifications of Cat8-1 and Cat8-2 would be necessary in order to elucidate if they are indeed activated via phosphorylation in $K$. phaffii, as observed for S. cerevisiae. 


\section{Conclusions}

The present study elucidated the requirement of the two Cat8 homologs Cat8-1 and Cat8-2 for activation of the ethanol assimilation pathway in $K$. phaffii, and their differential involvement in regulating the carnitine shuttle and the glyoxylate shunt, respectively. Phylogenetic analysis places Cat8-2 among the Sip4-like proteins. However, different to S. cerevisiae and $\mathrm{K}$. lactis, CAT8-2 expression is not regulated by the Cat8-1 TF, but autoregulated by its own gene product. Furthermore, CAT8-2 is repressed in the presence of Mig1-2 on glucose. Despite their transcriptional induction on both ethanol and methanol, no direct impact on methanol utilization genes could be observed in the Cat8 mutant strains. Interestingly, however, the CSRE motif, which was shown to be recognized by Cat8 and Sip4 in S. cerevisiae and $K$. lactis, was predicted by MatInspector to be present in around $90 \%$ of the total promoters in K. phaffii (Supplementary material 3). Contrary to what is described for cat $8 \Delta$ and sip4S in S. cerevisiae, knocking-out CAT8-1 and CAT8-2 also influences the ability of $K$. phaffii to tolerate osmotic stress and cell wall damaging agents (Supplementary figure S6). These results suggest that these two TFs might have additional targets and a broader role in $K$. phaffii, both of which remain to be investigated.

Supplementary Information The online version contains supplementary material available at https://doi.org/10.1007/s00294-021-01165-4.

Author contributions DB, DM, and BG contributed to the study conception and design. LS performed the phylogenetic analysis. DB created and characterized all Cat 8 mutant strains, while MM generated and cultivated the kinase mutant strains. SD characterized growth on acetate and performed the complementation experiments. The first draft of the manuscript was written by DB and BG and all authors commented on previous versions of the manuscript. All authors read and approved the final manuscript.

Funding Open access funding provided by University of Natural Resources and Life Sciences Vienna (BOKU). This work was supported by the Federal Ministry for Digital and Economic Affairs (bmwd), the Federal Ministry for Transport, Innovation and Technology (bmvit), the Styrian Business Promotion Agency SFG, the Standortagentur Tirol, Government of Lower Austria and ZIT-Technology Agency of the City of Vienna through the COMET (Competence Centers for Excellent Technologies) funding programme managed by the Austrian Research Promotion Agency FFG. Further support was received from the Austrian Science Fund FWF within the Doctoral Program 'Biomolecular Technology of Proteins' (BioTop, FWF W1224). MM received a scholarship from the Austrian-Slovakian action of the Austrian Agency for International Cooperation in Education and Research OeAD (ICM-2018-11997) financed by the Austrian Federal Ministry of Education, Science and Research (BMBWF).

Availability of data and materials All data generated or analyzed during this study are included in this published article and its supplementary information files.
Code availability Not applicable.

\section{Declarations}

Conflict of interest The authors declare that they have no conflict of interest.

Ethics approval Not applicable.

Consent to participate Not applicable.

Consent for publication Not applicable.

Open Access This article is licensed under a Creative Commons Attribution 4.0 International License, which permits use, sharing, adaptation, distribution and reproduction in any medium or format, as long as you give appropriate credit to the original author(s) and the source, provide a link to the Creative Commons licence, and indicate if changes were made. The images or other third party material in this article are included in the article's Creative Commons licence, unless indicated otherwise in a credit line to the material. If material is not included in the article's Creative Commons licence and your intended use is not permitted by statutory regulation or exceeds the permitted use, you will need to obtain permission directly from the copyright holder. To view a copy of this licence, visit http://creativecommons.org/licenses/by/4.0/.

\section{References}

Ata O, Prielhofer R, Gasser B, Mattanovich D, Calik P (2017) Transcriptional engineering of the glyceraldehyde-3-phosphate dehydrogenase promoter for improved heterologous protein production in Pichia pastoris. Biotechnol Bioeng 114:2319-2327

Barnett JA, Entian KD (2005) A history of research on yeasts 9: regulation of sugar metabolism. Yeast 22:835-894

Capella-Gutierrez S, Silla-Martinez JM, Gabaldon T (2009) trimAl: a tool for automated alignment trimming in large-scale phylogenetic analyses. Bioinformatics 25:1972-1973

Carlson M (1999) Glucose repression in yeast. Curr Opin Microbiol 2:202-207

Charbon G, Breunig KD, Wattiez R, Vandenhaute J, Noël-Georis I (2004) Key role of Ser562/661 in Snf1-dependent regulation of Cat8p in Saccharomyces cerevisiae and Kluyveromyces lactis. Mol Cell Biol 24:4083-4091

De Vit MJ, Waddle JA, Johnston M (1997) Regulated nuclear translocation of the Mig1 glucose repressor. Mol Biol Cell 8:1603-1618

Delic M, Mattanovich D, Gasser B (2013) Repressible promotersa novel tool to generate conditional mutants in Pichia pastoris. Microb Cell Fact 12:6

Engler C, Kandzia R, Marillonnet S (2008) A one pot, one step, precision cloning method with high throughput capability. PLoS ONE 3:e3647

Ergün BG, Gasser B, Mattanovich D, Çalık P (2019) Engineering of alcohol dehydrogenase 2 hybrid-promoter architectures in Pichia pastoris to enhance recombinant protein expression on ethanol. Biotechnol Bioeng 116:2674-2686

Ergün BG, Demir İ, Özdamar TH, Gasser B, Mattanovich D, Çalık P (2020) Engineered deregulation of expression in yeast with designed hybrid-promoter architectures in coordination with discovered master regulator transcription factor. Adv Biosyst 4:e1900172 
Foley G, Sützl L, D’Cunha SA, Gillam EMJ, Bodén M (2019) SeqScrub: a web tool for automatic cleaning and annotation of FASTA file headers for bioinformatic applications. Biotechniques 67:50-54

Gasser B, Prielhofer R, Marx H, Maurer M, Nocon J, Steiger M, Puxbaum V, Sauer M, Mattanovich D (2013) Pichia pastoris: protein production host and model organism for biomedical research. Future Microbiol 8:191-208

Gassler T, Heistinger L, Mattanovich D, Gasser B, Prielhofer R (2019) CRISPR/Cas9-mediated homology directed genome editing in Pichia pastoris. Methods Mol Biol 1923:211-225

Georis I, Krijger JJ, Breunig KD, Vandenhaute J (2000) Differences in regulation of yeast gluconeogenesis revealed by Cat8p-independent activation of $P C K 1$ and $F B P 1$ genes in Kluyveromyces lactis. Mol Gen Genet 264:193-203

Guindon S, Dufayard JF, Lefort V, Anisimova M, Hordijk W, Gascuel O (2010) New algorithms and methods to estimate maximumlikelihood phylogenies: assessing the performance of PhyML 3.0. Syst Biol 59:307-321

Hardie DG, Carling D, Carlson M (1998) The AMP-activated/SNF1 protein kinase subfamily: metabolic sensors of the eukaryotic cell? Annu Rev Biochem 67:821-855

Hartner FS, Glieder A (2006) Regulation of methanol utilisation pathway genes in yeasts. Microb Cell Fact 5:39

Haurie V, Perrot M, Mini T, Jenö P, Sagliocco F, Boucherie H (2001) The transcriptional activator Cat8p provides a major contribution to the reprogramming of carbon metabolism during the diauxic shift in Saccharomyces cerevisiae. J Biol Chem 276:76-85

Hedbacker K, Carlson M (2008) SNF1/AMPK pathways in yeast. Front Biosci 13:2408-2420

Hedges D, Proft M, Entian KD (1995) CAT8, a new zinc cluster-encoding gene necessary for derepression of gluconeogenic enzymes in the yeast Saccharomyces cerevisiae. Mol Cell Biol 15:1915-1922

Hiesinger M, Roth S, Meissner E, Schuller HJ (2001) Contribution of Cat8 and Sip4 to the transcriptional activation of yeast gluconeogenic genes by carbon source-responsive elements. Curr Genet 39:68-76

Katoh K, Standley DM (2013) MAFFT Multiple Sequence Alignment Software Version 7: improvements in performance and usability. Mol Biol Evol 30:772-780

Kranthi BV, Kumar HR, Rangarajan PN (2010) Identification of Mxr1p-binding sites in the promoters of genes encoding dihydroxyacetone synthase and peroxin 8 of the methylotrophic yeast Pichia pastoris. Yeast 27:705-711

Kratzer S, Schüller HJ (1997) Transcriptional control of the yeast acetyl-CoA synthetase gene, ACS1, by the positive regulators CAT8 and ADR1 and the pleiotropic repressor UME6. Mol Microbiol 26:631-641

Landes N, Gasser B, Vorauer-Uhl K, Lhota G, Mattanovich D, Maurer $\mathrm{M}$ (2016) The vitamin-sensitive promoter $\mathrm{P}_{T H 11}$ enables predefined autonomous induction of recombinant protein production in Pichia pastoris. Biotechnol Bioeng 113:2633-2643

Lesage P, Yang X, Carlson M (1996) Yeast SNF1 protein kinase interacts with SIP4, a C6 zinc cluster transcriptional activator: a new role for SNF1 in the glucose response. Mol Cell Biol 16:1921-1928

Lin-Cereghino GP, Godfrey L, de la Cruz BJ, Johnson S, Khuongsathiene S, Tolstorukov I, Yan M, Lin-Cereghino J, Veenhuis M, Subramani S, Cregg JM (2006) Mxr1p, a key regulator of the methanol utilization pathway and peroxisomal genes in Pichia pastoris. Mol Cell Biol 26:883-897

Mehlgarten C, Krijger JJ, Lemnian I, Gohr A, Kasper L, Diesing AK, Grosse I, Breunig KD (2015) Divergent evolution of the transcriptional network controlled by Snf1-Interacting protein Sip4 in budding yeasts. PLoS ONE 10:e139464
Monteiro PT, Oliveira J, Pais P, Antunes M, Palma M, Cavalheiro M, Galocha M, Godinho CP, Martins LC, Bourbon N, Mota MN, Ribeiro RA, Viana R, Sá-Correia I, Teixeira MC (2020) YEASTRACT+: a portal for cross-species comparative genomics of transcription regulation in yeasts. Nucleic Acids Res 48:D642-D649

Palmieri L, Lasorsa FM, De Palma A, Palmieri F, Runswick MJ, Walker JE (1997) Identification of the yeast $A C R 1$ gene product as a succinate-fumarate transporter essential for growth on ethanol or acetate. FEBS Lett 417:114-118

Prielhofer R, Barrero JJ, Steuer S, Gassler T, Zahrl R, Baumann K, Sauer M, Mattanovich D, Gasser B, Marx H (2017) GoldenPiCS: a Golden Gate-derived modular cloning system for applied synthetic biology in the yeast Pichia pastoris. BMC Syst Biol 11:123

Prielhofer R, Cartwright SP, Graf AB, Valli M, Bill RM, Mattanovich D, Gasser B (2015) Pichia pastoris regulates its gene-specific response to different carbon sources at the transcriptional, rather than the translational, level. BMC Genomics 16:167

Qi K, Zhong JJ, Xia XX (2014) Triggering respirofermentative metabolism in the crabtree-negative yeast Pichia guilliermondii by disrupting the CAT8 gene. Appl Environ Microbiol 80:3879-3887

Rahner A, Schöler A, Martens E, Gollwitzer B, Schüller HJ (1996) Dual influence of the yeast Cat1p $(\operatorname{Snf} 1 \mathrm{p})$ protein kinase on carbon source-dependent transcriptional activation of gluconeogenic genes by the regulatory gene CAT8. Nucleic Acids Res 24:2331-2337

Ramirez MA, Lorenz MC (2009) The transcription factor homolog CTF1 regulates $\{$ beta $\}$-oxidation in Candida albicans. Eukaryot Cell 8:1604-1614

Randez-Gil F, Bojunga N, Proft M, Entian KD (1997) Glucose derepression of gluconeogenic enzymes in Saccharomyces cerevisiae correlates with phosphorylation of the gene activator Cat8p. Mol Cell Biol 17:2502-2510

Rodicio R, López ML, Cuadrado S, Cid AF, Redruello B, Moreno F, Heinisch JJ, Hegewald AK, Breunig KD (2008) Differential control of isocitrate lyase gene transcription by non-fermentable carbon sources in the milk yeast Kluyveromyces lactis. FEBS Lett 582:549-557

Roth S, Kumme J, Schüller HJ (2004) Transcriptional activators Cat8 and Sip4 discriminate between sequence variants of the carbon source-responsive promoter element in the yeast Saccharomyces cerevisiae. Curr Genet 45:121-128

Ruchala J, Kurylenko OO, Soontorngun N, Dmytruk KV, Sibirny AA (2017) Transcriptional activator Cat8 is involved in regulation of xylose alcoholic fermentation in the thermotolerant yeast Ogataea (Hansenula) polymorpha. Microb Cell Fact 16:36

Sahu U, Rangarajan PN (2016) Regulation of acetate metabolism and acetyl Co-A synthetase 1 (ACS1) expression by methanol expression regulator $1(\mathrm{Mxr} 1 \mathrm{p})$ in the methylotrophic yeast Pichia pastoris. J Biol Chem 291:3648-3657

Schmalix W, Bandlow W (1993) The ethanol-inducible YAT1 gene from yeast encodes a presumptive mitochondrial outer carnitine acetyltransferase. J Biol Chem 268:27428-27439

Schüller HJ (2003) Transcriptional control of nonfermentative metabolism in the yeast Saccharomyces cerevisiae. Curr Genet 43:139-160

Shen W, Kong C, Xue Y, Liu Y, Cai M, Zhang Y, Jiang T, Zhou X, Zhou M (2016) Kinase screening in Pichia pastoris identified promising targets involved in cell growth and alcohol oxidase 1 promoter $\left(\mathrm{P}_{A O X I}\right)$ regulation. PLoS ONE 11:e0167766

Shi L, Wang X, Wang J, Zhang P, Qi F, Cai M, Zhang Y, Zhou X (2018) Transcriptome analysis of $\Delta m i g 1 \Delta m i g 2$ mutant reveals their roles in methanol catabolism, peroxisome biogenesis and autophagy in methylotrophic yeast Pichia pastoris. Genes Genomics 40:399-412 
Soontorngun N, Larochelle M, Drouin S, Robert F, Turcotte B (2007) Regulation of gluconeogenesis in Saccharomyces cerevisiae is mediated by activator and repressor functions of Rds2. Mol Cell Biol 27:7895-7905

Stemple CJ, Davis MA, Hynes MJ (1998) The facC gene of Aspergillus nidulans encodes an acetate-inducible carnitine acetyltransferase. J Bacteriol 180:6242-6251

Tachibana C, Yoo JY, Tagne JB, Kacherovsky N, Lee TI, Young ET (2005) Combined global localization analysis and transcriptome data identify genes that are directly coregulated by Adr1 and Cat8. Mol Cell Biol 25:2138-2146

Todd RB, Andrianopoulos A (1997) Evolution of a fungal regulatory gene family: the $\mathrm{Zn}(\mathrm{II}) 2 \mathrm{Cys} 6$ binuclear cluster DNA binding motif. Fungal Genet Biol 21:388-405

Todd RB, Kelly JM, Davis MA, Hynes MJ (1997) Molecular characterization of mutants of the acetate regulatory gene facB of Aspergillus nidulans. Fungal Genet Biol 22:92-102

Todd RB, Andrianopoulos A, Davis MA, Hynes MJ (1998) FacB, the Aspergillus nidulans activator of acetate utilization genes, binds dissimilar DNA sequences. EMBO J 17:2042-2054

Toussaint M, Conconi A (2006) High-throughput and sensitive assay to measure yeast cell growth: a bench protocol for testing genotoxic agents. Nat Protoc 1:1922-1928

Turcotte B, Liang XB, Robert F, Soontorngun N (2010) Transcriptional regulation of nonfermentable carbon utilization in budding yeast. FEMS Yeast Res 10:2-13
Valli M, Tatto NE, Peymann A, Gruber C, Landes N, Ekker H, Thallinger GG, Mattanovich D, Gasser B, Graf AB (2016) Curation of the genome annotation of Pichia pastoris (Komagataella phaffii) CBS7435 from gene level to protein function. FEMS Yeast Res 16:fow051

Vincent O, Carlson M (1998) Sip4, a Snf1 kinase-dependent transcriptional activator, binds to the carbon source-responsive element of gluconeogenic genes. EMBO J 17:7002-7008

Vincent O, Carlson M (1999) Gal83 mediates the interaction of the Snf1 kinase complex with the transcription activator Sip4. EMBO J 18:6672-6681

Vincent O, Kuchin S, Hong SP, Townley R, Vyas VK, Carlson M (2001) Interaction of the Srb10 kinase with Sip4, a transcriptional activator of gluconeogenic genes in Saccharomyces cerevisiae. Mol Cell Biol 21:5790-5796

Walther K, Schüller HJ (2001) Adr1 and Cat8 synergistically activate the glucose-regulated alcohol dehydrogenase gene $A D H 2$ of the yeast Saccharomyces cerevisiae. Microbiol 147:2037-2044

Wang J, Wang X, Shi L, Qi F, Zhang P, Zhang Y, Zhou X, Song Z, Cai M (2017) Methanol-independent protein expression by AOXI promoter with trans-acting elements engineering and glucoseglycerol-shift induction in Pichia pastoris. Sci Rep 7:41850

Publisher's Note Springer Nature remains neutral with regard to jurisdictional claims in published maps and institutional affiliations. 